The Astrophysical Journal, 689:436-460, 2008 December 10

(C) 2008. The American Astronomical Society. All rights reserved. Printed in U.S.A.

\title{
KECK LASER GUIDE STAR ADAPTIVE OPTICS MONITORING OF 2MASS J15344984-2952274AB: FIRST DYNAMICAL MASS DETERMINATION OF A BINARY T DWARF ${ }^{1,2}$
}

\author{
Michael C. Liu, ${ }^{3,4,5}$ Trent J. Dupuy, ${ }^{3}$ and Michael J. Ireland ${ }^{6,7}$ \\ Received 2007 December 20; accepted 2008 July 1
}

\begin{abstract}
We present multiepoch imaging of the T5.0+T5.5 binary 2MASS J15344984-2952274AB obtained with the Keck laser guide star adaptive optics system. Combined with archival HST imaging, our total data span $\sim 50 \%$ of the orbital period. We use a Markov chain Monte Carlo analysis to determine a period of $15.1_{-1.6}^{+2.3} \mathrm{yr}$ and a total mass of $0.056 \pm 0.003 M_{\odot}\left(59 \pm 3 M_{\mathrm{J}}\right)$. This is the first field binary for which both components are confirmed to be substellar. This is also the coolest and lowest mass binary with a dynamical mass to date. Using evolutionary models and accounting for the measurement covariances, we derive an age of $0.78 \pm 0.09 \mathrm{Gyr}$ and a mass ratio of $0.936_{-0.008}^{+0.012}$. The relatively youthful age is consistent with the low tangential velocity of this system. For the individual components, we find $T_{\text {eff }}=1028 \pm 17$ and $978 \pm 17 \mathrm{~K}$ and masses of $0.0287 \pm 0.0016 M_{\odot}\left(30.1 \pm 1.7 M_{\mathrm{J}}\right)$ and $0.0269 \pm$ $0.0016 M_{\odot}\left(28.2 \pm 1.7 M_{\mathrm{J}}\right)$. These values generally agree with previous studies of $\mathrm{T}$ dwarfs and affirm current theoretical models. However, (1) the temperatures are about $100 \mathrm{~K}$ cooler than derived for similar objects and suggest that the representative ages of field brown dwarfs may be overestimated. Similarly, (2) the H-R diagram positions are discrepant with current models and taken at face value would overestimate the masses. While this may arise from errors in the luminosities and/or radii predicted by evolutionary models, the likely cause is a modest $(\approx 100 \mathrm{~K})$ overestimate in temperature determined from model atmospheres. We elucidate future tests of theory as the sample of dynamical masses grows. In particular, we suggest that low-mass field binaries with dynamical masses ("mass benchmarks") can serve as reference points for $T_{\text {eff }}$ and $\log g$ to constrain atmospheric models, as good as or even better than single brown dwarfs with age estimates ("age benchmarks").
\end{abstract}

Subject headings: binaries: close — binaries: visual — infrared: stars — stars: fundamental parameters stars: low-mass, brown dwarfs — techniques: high angular resolution

\section{INTRODUCTION}

Over about the past decade, the parameter space of traditional stellar astrophysics has been greatly expanded with the discovery and characterization of brown dwarfs, objects that for most of their lifetimes are colder and less luminous than main-sequence stars. Despite ample progress in finding and characterizing brown dwarfs, very few direct measurements of their physical properties have been made so far. In particular, dynamical masses for brown dwarfs are sorely needed to test the theoretical models over a wide range of parameter space. In comparison to the $>100$ binary stars with direct mass determinations, dynamical masses have been measured for only a handful of objects clearly below the stellar/ substellar boundary:

\footnotetext{
${ }^{1}$ Most of the data presented herein were obtained at the W. M. Keck Observatory, which is operated as a scientific partnership among the California Institute of Technology, the University of California, and the National Aeronautics and Space Administration. The Observatory was made possible by the generous financial support of the W. M. Keck Foundation.

${ }_{2}^{2}$ Based on observations made with the NASA/ESA Hubble Space Telescope, obtained from the Data Archive at the Space Telescope Science Institute, which is operated by the Association of Universities for Research in Astronomy, Inc., under NASA contract NAS 5-26555.

${ }^{3}$ Institute for Astronomy, University of Hawaii, Honolulu, HI 96822; mliu@ifa.hawaii.edu.

4 Alfred P. Sloan Research Fellow.

5 Visiting Astronomer at the Infrared Telescope Facility, which is operated by the University of Hawaii under Cooperative Agreement NNX08AE38A with the National Aeronautics and Space Administration, Science Mission Directorate, Planetary Astronomy Program.

${ }^{6}$ Division of Geological and Planetary Sciences, California Institute of Technology, Pasadena, CA 91125.

7 School of Physics, University of Sydney, Sydney NSW 2006, Australia.
}

1. The secondary component of the M8.5+M9 visual binary Gl 569Bab, which itself is a companion to a young $(\approx 100-300 \mathrm{Myr})$ field M2.5 dwarf (Martín et al. 2000; Lane et al. 2001; Kenworthy et al. 2001; Zapatero Osorio et al. 2004).

2. The very young $(\sim 1 \mathrm{Myr})$ eclipsing M6.5+M6.5 binary brown dwarf 2MASS J05352184-0546085 (Stassun et al. 2006).

3. The secondary component of the GJ 802AB system, where the secondary has an estimated spectral type of L5 and the primary is an M5.5+M5.5 spectroscopic binary (Pravdo et al. 2006; Lloyd et al. 2006; Ireland et al. 2008).

In addition, GJ 569Ba itself may be an unresolved binary brown dwarf (Simon et al. 2006), and the secondary component of the L0+L1.5 binary 2MASSW J0746425+2000321 AB, which appears to be an old ( $21 \mathrm{Gyr}$ ) field system, has a mass near the stellar/ substellar boundary (Reid et al. 2001; Bouy et al. 2004; Gizis \& Reid 2006).

About 100 ultracool visual binaries are known, ${ }^{8}$ found with high angular resolution imaging surveys conducted by Hubble Space Telescope (HST; e.g., Reid et al. 2001; Bouy et al. 2003; Gizis et al. 2003; Burgasser et al. 2003b, 2006c) and ground-based adaptive optics (AO) imaging (e.g., Close et al. 2003; Burgasser et al. 2005a; Liu et al. 2006; Reid et al. 2008; M. C. Liu et al. 2008, in preparation). Only $\approx 10 \%$ of these are binaries composed of the coldest class of brown dwarf, the $\mathrm{T}$ dwarfs. $\mathrm{T}$ dwarfs are distinguished by their very red optical colors arising from pressurebroadened alkali resonance lines and very blue near-IR colors from strong $\mathrm{CH}_{4}, \mathrm{H}_{2} \mathrm{O}$, and collision-induced $\mathrm{H}_{2}$ absorption (e.g., Oppenheimer et al. 1995; Geballe et al. 2002; Kirkpatrick 2005).

\footnotetext{
8 We follow the popular convention that "ultracool" refers to objects of (integrated light) spectral type M6 or later.
} 
These are the lowest luminosity and coolest objects directly detected outside of our solar system, with bolometric luminosities $\left(L_{\text {bol }}\right)$ of $\lesssim 10^{-4.5} L_{\odot}$ and effective temperatures $\left(T_{\text {eff }}\right)$ of $\approx 600-$ 1300 K (e.g., Vrba et al. 2004; Golimowski et al. 2004a; Burgasser et al. 2006a; Leggett et al. 2007a; Warren et al. 2007; Delorme et al. 2008). As such, analyzing their physical properties is an important pathway to understanding the properties of gas giant extrasolar planets.

The subject of this paper is the T dwarf 2MASS J153449842952274AB, hereinafter 2MASS J1534-2952AB, which has an integrated-light infrared spectral type of T5 (Burgasser et al. 2002, 2006b). ${ }^{9}$ This object was first resolved as a $0.065^{\prime \prime}$ binary in 2000 August in HST WFPC2 imaging (Burgasser et al. 2003b). Among known visual ultracool binaries in the field, this system has the shortest estimated orbital period, only $4 \mathrm{yr}$ (see compilation in Burgasser et al. 2007). In combination with its very high quality parallax measurement of $73.6 \pm 1.2$ mas (Tinney et al. 2003) and the fact that its two components are nearly equal magnitude (indicating nearly equal masses), this system is a very appealing object for astrometric monitoring.

Laser guide star (LGS) AO provides a powerful tool for high angular resolution studies of brown dwarf binaries. Through resonant scattering off the sodium layer at $\sim 90 \mathrm{~km}$ altitude in the Earth's atmosphere, sodium LGS systems create an artificial star bright enough to serve as a wave front reference for AO correction (Foy \& Labeyrie 1985; Thompson \& Gardner 1987; Happer et al. 1994). Thus, most of the sky can be made accessible to near diffraction-limited IR imaging from the largest existing groundbased telescopes. We have previously used Keck LGS AO to discover that the nearby L dwarf Kelu-1 is a binary system (Liu \& Leggett 2005) and to identify the novel L+T binary SDSS J1534+ $1615 \mathrm{AB}$ (Liu et al. 2006). In regards to dynamical mass determinations, ground-based telescopes equipped with LGS AO can provide the necessary long-term platforms for synoptic monitoring of visual binaries, especially where the required amount of observing time at each epoch is relatively modest but many epochs are needed, in contrast to $H S T$ where target acquisition can be slow and monitoring a populous sample over many epochs is quite telescope time intensive.

We present here the results of multiepoch imaging of 2MASS J1534-2952AB, observed as part of our ongoing high angular resolution study of ultracool binaries using LGS AO. Section 2 presents our Keck LGS AO observations and (re)analysis of archival HST imaging. Section 3 presents the resolved photometric properties of the binary and fitting of the orbit using a Markov chain Monte Carlo method. Section 4 compares the resulting total mass against evolutionary models, and $\S 5$ summarizes our findings. Those readers interested solely in the results can focus on $\S \S 4$ and 5.

\section{OBSERVATIONS}

\subsection{Keck $L G S A O$}

We imaged 2MASS J1534-2952AB from 2005-2008 using the sodium LGS AO system of the $10 \mathrm{~m}$ Keck II Telescope on Mauna Kea, Hawaii (Wizinowich et al. 2006; van Dam et al. 2006). Conditions were photometric for all the runs. We used the

\footnotetext{
${ }^{9}$ Burgasser et al. (2006b) report integrated-light spectral types of both T5 and T5.5 for 2MASS J1534-2952AB based on a spectrum obtained with the $\mathrm{CTIO} / \mathrm{OSIRIS}$ instrument. Examination of their spectrum by us finds that T5 is the correct typing. Also, a new near-IR spectrum obtained with the IRTF/SpeX spectrograph confirms an integrated-light spectral type of T5 $(\S 2.3)$.
}

facility IR camera NIRC2 with its narrow field-of-view camera, which produces a $10.2^{\prime \prime} \times 10.2^{\prime \prime}$ field of view. Setup times for the telescope to slew to the science targets and for the LGS AO system to be fully operational ranged from 7 to 20 minutes, with an average of 12 minutes (e.g., Liu 2006).

The LGS provided the wave front reference source for AO correction, with the exception of tip-tilt motion. The LGS brightness, as measured by the flux incident on the $\mathrm{AO}$ wave front sensor, was equivalent to a $V \approx 9.2-10.3 \mathrm{mag}$ star. Tip-tilt aberrations and quasi-static changes in the image of the LGS as seen by the wave front sensor were measured contemporaneously with a second, lower bandwidth wave front sensor monitoring the $R=$ 16.2 mag field star USNO-B1.0 0601-0344964 (Monet et al. 2003), located $31^{\prime \prime}$ away from 2MASS J1534-2952AB.

At each epoch, 2MASS J1534-2952AB was imaged in filters covering the standard $2.2 \mu \mathrm{m}$ atmospheric window from the Mauna Kea Observatories (MKO) filter consortium (Simons \& Tokunaga 2002; Tokunaga et al. 2002). Our initial observations in 2005 April were carried out with the $K^{\prime}(2.12 \mu \mathrm{m})$ filter to minimize the thermal background from the AO system, which is kept at ambient temperature. Subsequent runs employed the $K(2.20 \mu \mathrm{m})$ or $K_{s}(2.15 \mu \mathrm{m})$ filters. Hereinafter, for brevity we refer to all these data simply as $K$-band observations.

On each observing run, we typically obtained a series of dithered $K$-band images, offsetting the telescope by a few arcseconds between each one to two images. The sodium laser beam was pointed at the center of the NIRC2 field of view for all observations. In 2005 April, we also obtained images with the MKO $J(1.25 \mu \mathrm{m})$ and $H(1.64 \mu \mathrm{m})$ filters. In 2008 April, we also obtained images with the $\mathrm{CH}_{4} s$ filter, which has a central wavelength of $1.592 \mu \mathrm{m}$ and a width of $0.126 \mu \mathrm{m}$; this filter is positioned around the $H$-band flux peak in the spectra of mid/late T dwarfs.

The images were reduced in a standard fashion. We constructed flat fields from the differences of images of the telescope dome interior with and without continuum lamp illumination. Then we created a master sky frame from the median average of the biassubtracted, flat-fielded images and subtracted it from the individual images. Images were registered and stacked to form a final mosaic, although all the results described here were based on analysis of the individual images. Outlier images with much poorer FWHM and/or Strehl ratios were excluded from the analysis. Instrumental optical distortion was corrected based on analysis by B. Cameron (2007, private communication) of images of a precisely machined pinhole grid located at the first focal plane of NIRC2. The $1 \sigma$ residuals of the pinhole images after applying this distortion correction are at the 0.6 mas level over the detector field of view. Since the binary separation and the imaging dither steps are small, the effect of the distortion correction is minor, smaller than our final measurement errors.

Table 1 compiles the details of our observations, and Figure 1 presents our Keck LGS data. Full widths at half maxima (FWHMs) and Strehl ratios were determined from two field stars located $\approx 5^{\prime \prime}-6^{\prime \prime}$ from 2MASS J1534-2952AB. The tabulated errors on the FWHMs and Strehl ratios are the standard deviation of these quantities as measured from the individual images.

To measure the flux ratios and relative positions of 2MASS J1534-2952AB's two components, we mostly used the two aforementioned nearby field stars, observed simultaneously with 2MASS J1534-2952AB on NIRC2. These stars provided an excellent measurement of the instantaneous point-spread function (PSF). We empirically modeled the PSF using the Starfinder software package (Diolaiti et al. 2000), which is designed for analysis of blended AO images. For the 2008 January data, we employed 
TABLE 1

KeCK LGS AO ObSERVATIONS

\begin{tabular}{|c|c|c|c|c|c|c|c|}
\hline $\begin{array}{l}\text { Date } \\
\text { (UT) }\end{array}$ & Filter $^{\mathrm{a}}$ & Air Mass & $\begin{array}{l}\text { FWHM } \\
\text { (mas) }\end{array}$ & Strehl Ratio & $\begin{array}{l}\text { Separation } \\
\text { (mas) }\end{array}$ & $\begin{array}{c}\text { Position Angle } \\
\text { (deg) }\end{array}$ & $\Delta \mathrm{mag}$ \\
\hline \multirow[t]{3}{*}{ 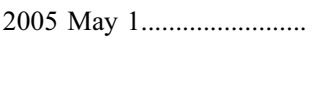 } & $J$ & 1.66 & $102 \pm 12$ & $0.020 \pm 0.002$ & $211.3 \pm 1.5(1.5)$ & $14.1 \pm 0.3(0.3)$ & $0.163 \pm 0.014$ \\
\hline & $H$ & 1.63 & $86 \pm 6$ & $0.047 \pm 0.006$ & $211.7 \pm 0.8(0.8)$ & $13.86 \pm 0.15(0.13)$ & $0.286 \pm 0.011$ \\
\hline & $K^{\prime}$ & 1.61 & $88 \pm 6$ & $0.101 \pm 0.012$ & $212.4 \pm 1.1(1.0)$ & $14.0 \pm 0.2(0.2)$ & $0.278 \pm 0.021$ \\
\hline 2006 May $5 .$. & $K_{s}$ & 1.56 & $64 \pm 3$ & $0.210 \pm 0.014$ & $190.6 \pm 0.3(0.2)$ & $15.43 \pm 0.12(0.09)$ & $0.282 \pm 0.010$ \\
\hline 2007 Mar 26. & $K$ & 1.56 & $82 \pm 3$ & $0.151 \pm 0.016$ & $158.0 \pm 0.6(0.6)$ & $17.5 \pm 0.2(0.19)$ & $0.287 \pm 0.012$ \\
\hline 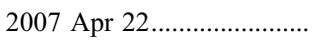 & $K_{s}$ & 1.57 & $67 \pm 5$ & $0.20 \pm 0.03$ & $153.7 \pm 0.4(0.3)$ & $17.53 \pm 0.13(0.10)$ & $0.269 \pm 0.010$ \\
\hline 2008 Jan $15 \ldots$ & $K_{s}$ & 2.05 & $100 \pm 3$ & $0.074 \pm 0.002$ & $114.4 \pm 1.1(1.1)$ & $21.5 \pm 0.9(0.9)$ & $0.27 \pm 0.06$ \\
\hline \multirow[t]{2}{*}{ 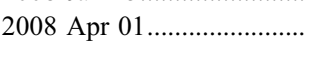 } & $K_{s}$ & 1.55 & $87 \pm 4$ & $0.095 \pm 0.018$ & $102.5 \pm 0.7(0.7)$ & $21.1 \pm 0.7(0.7)$ & $0.25 \pm 0.04$ \\
\hline & $\mathrm{CH}_{4} s$ & 1.58 & $78 \pm 7$ & $0.048 \pm 0.018$ & $102.0 \pm 0.4(0.4)$ & $20.4 \pm 1.5(1.5)$ & $0.21 \pm 0.04$ \\
\hline
\end{tabular}

a All photometry on the MKO system.

b The tabulated errors are computed by appropriately combining in quadrature (1) the instrumental measurements from fitting the images of the binary and (2) the overall uncertainties in the NIRC2 pixel scale and orientation. The errors in parentheses represent the instrumental errors alone. See $\S 2$ for details.

a different procedure, fitting analytic PSFs comprising multiple elliptical Gaussians to model the binary images. These data were taken at much higher air mass than all the other data. Because of the larger atmospheric dispersion and the different colors of the field stars relative to 2MASS J1534-2952AB, PSF fitting produced less accurate results than the analytic approach, as determined by the artificial binary tests described below. For every image, we fitted for the fluxes and positions of the two components and then computed the flux ratio, separation, and position angle (P.A.) of the binary. The averages of the results were adopted as the final measurements. Overall, our PSF fitting produced very high quality relative measurements, with errors of order $1 \%$ for the flux ratios, 0.05 pixels for the binary separation, and $0.2^{\circ}$ for the P.A. Note that the latter two values account only for the internal
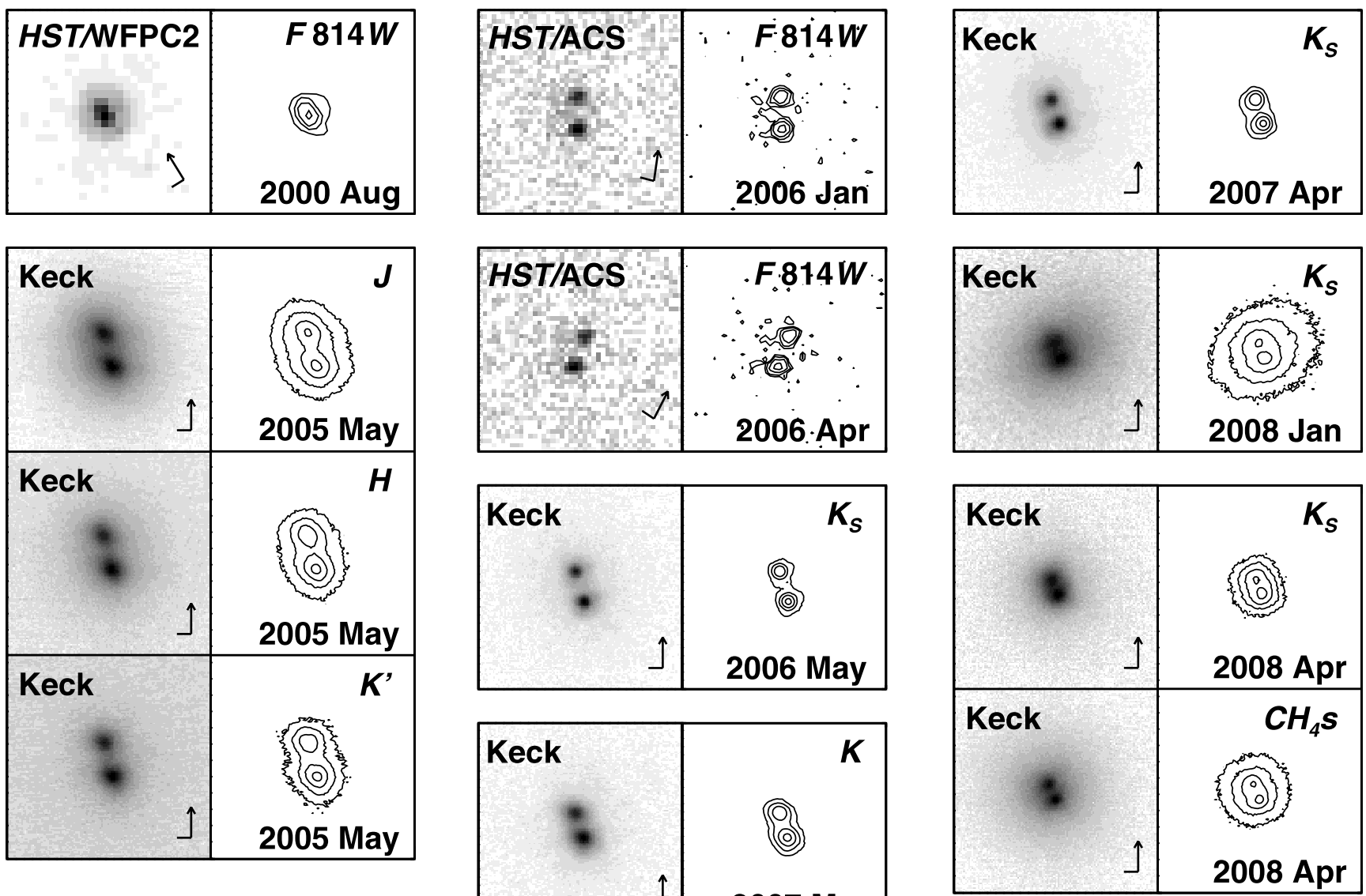

FIG. 1.- Images of 2MASS J1534-2952AB from HST WFPC2, Keck LGS, and HST ACS, arranged chronologically in each column. Each image is 1.24" (16.8 AU) on a side, with the orientation indicated by the compass roses. Note that the sky directions for the ACS images are not orthogonal, due to optical distortion in this instrument. (We chose not to rotate the HST images to the orientation of the Keck image for this figure, but instead to display the actual images so as to preserve the quality

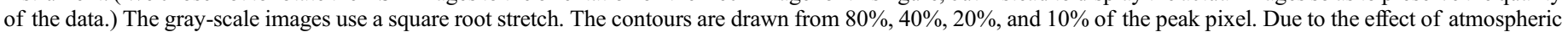
dispersion, the Keck LGS $J$ - and $H$-band images are slightly elongated in the vertical direction (which coincided with the elevation axis for these observations). 
instrumental measurements and do not include the errors on the astrometric calibration of NIRC2, which we include below.

In order to gauge the accuracy of our measurements, we created myriad artificial binary stars from images of the two PSF stars. One PSF star was used to create artificial binaries, and the other was used as the single PSF for fitting the components. For data at each epoch, Starfinder was applied to the artificial binaries with similar separations and flux ratios as 2MASS J15342952AB. These simulations showed that any systematic offsets in our fitting code are very small, well below the random errors, and that the random errors are accurate. In cases where the rms measurement errors from the artificial binaries were larger than those from the 2MASS J1534-2952AB measurements, we conservatively adopted the larger errors. ${ }^{10}$

To convert the instrumental measurements of the binary separation and P.A. into celestial units, we used a weighted average of the calibration from Pravdo et al. (2006) with a pixel scale of $9.963 \pm$ 0.011 mas pixel $^{-1}$ and an orientation for the detector's $+y$-axis of $-0.13^{\circ} \pm 0.07^{\circ}$ east of north. These values agree well with Keck Observatory's notional calibration of $9.942 \pm 0.05$ mas pixel ${ }^{-1}$ and $0.0^{\circ} \pm 0.5^{\circ}$, as well as the $9.961 \pm 0.007$ mas pixel $^{-1}$ and $-0.015^{\circ} \pm 0.134^{\circ}$ reported by Konopacky et al. (2007). Also, comparison of NIRC2 images of M92 to astrometrically calibrated HST Advanced Camera for Surveys (ACS) Wide Field Camera images gives a pixel scale for NIRC2 that agrees to better than 1 part in $10^{-3}$ with our values (J. Anderson 2007, private communication).

Finally, we must consider the effect of atmospheric refraction. Because of the southern declination of 2MASS J1534-2952AB, all of our Keck observations were necessarily undertaken at significant air mass $(>1.55)$. Because the two components of the binary do not have exactly the same spectral types ( $\S 3.1)$, the observed positions on the sky are subject to slightly different amounts of differential chromatic refraction (DCR). We computed the expected shift in the relative astrometry at each epoch using the prescriptions of Monet et al. (1992) for the DCR offset and Stone (1984) for the refractive index of dry air. We assumed a fiducial temperature of $275 \mathrm{~K}$ and pressure of 608 mbar for conditions on Mauna Kea (Cohen \& Cromer 1988). We computed the effective wavelengths for spectral types of T5.0 and T5.5 for the two components using all available spectra of these subclasses contained in the SpeX Prism Spectral Library (from Burgasser et al. 2004; Chiu et al. 2006; Looper et al. 2007) and the appropriate filter response curve. ${ }^{11}$ Note that because of the unusual spectra of $\mathrm{T}$ dwarfs, the effective wavelengths of the secondary component are actually bluer in $H$ and $K$ bands and redder only in $J$ band compared to the primary component. Given the fact that the secondary is mostly north of the primary at all of our Keck epochs, DCR causes the separation of the binary to appear slightly smaller at $H$ and $K$ bands and slightly larger at $J$ band compared to the true

\footnotetext{
${ }^{10}$ We also experimented with directly fitting the binary data by themselves, without using other stars as the PSF. This approach was similar to our previous analyses (Liu \& Leggett 2005; Liu et al. 2006), namely, we fitted the images of the binary with either (1) an analytic model of the PSF as the sum of elliptical Gaussians or (2) an empirical model derived iteratively using a Starfinder-based code. These were also tested against images of simulated binaries. As before, we found that the Starfinder measurements were somewhat better compared to the analytical fits for the $K$-band data, and the multi-Gaussian fitting was better for the $J$ - and $H$-band data. Overall, fitting the binary images by themselves produced very good astrometry, with internal errors (i.e., without the uncertainty in the absolute NIRC 2 astrometric calibration) of about $3 \%$ for the flux ratio, 0.1 pixels for the separation, and $0.3^{\circ}$ for the P.A., but slightly worse than our fits using field stars as PSFs. The exception was the 2008 January data, as described in the text. ${ }^{11} \mathrm{MKO}$ filter curves are available at ftp://ftp.jach.hawaii.edu/pub/ukirt/ skl/ filters.
}

position as would be observed at zenith. The amplitude of the DCR effect is about 0.3 mas, much smaller than the measurement errors at most (but not all) of the Keck epochs. However, the effect is a systematic one so we correct the relative astrometry of the two components based on our calculations.

Table 1 presents the final resulting measurements from our Keck LGS data. For the 2005 April data set, all three filters give astrometry consistent within the measurement errors; we use only the $H$-band results in the orbit fitting discussed below. In the table and in our orbit fitting $(\S 3.3)$, we take care to discriminate between the instrumental errors (namely, those that arise solely from fitting the binary images) and the overall astrometric calibration of NIRC2; thus, any future refinements in the latter can be readily applied to our measurements.

\subsection{HST}

\subsubsection{WFPC2 Planetary Camera}

The two components of 2MASS J1534-2952AB are only barely resolved in the HST WFPC2 F814W discovery images from 2000 August. Therefore, to determine their relative positions and fluxes, we must model the images using the sum of two blended PSFs. The PSF of WFPC2's Planetary Camera (PC) is undersampled $(\mathrm{FWHM}=1.7$ pixels for $\mathrm{F} 814 \mathrm{~W})$; this makes any empirical determination of the PSF difficult without PSFs sampled at many subpixel locations. Moreover, Anderson \& King (2003) found that the WFPC2 PSF varies significantly over the detector due to geometric distortion, making it impossible to construct a reliable empirical PSF from other stars in the same image, even if there are enough to sample many subpixel locations. The original analysis by Burgasser et al. (2003b) employed a hybrid Gaussian/ empirical PSF to fit for the binary parameters with resulting uncertainties of \pm 7 mas in separation and $\pm 9^{\circ}$ in P.A. The astrometry from the WFPC2 discovery epoch is obviously very important to the orbit determination, so we undertook our own analysis with a more accurate PSF model to improve the precision of the binary parameters.

We used the Tiny Tim software package (Krist 1995) to create model PSFs for the WFPC2 images. We generated 5 times supersampled PSFs that included the effects of (1) variation with position on the detector; (2) broadband wavelength dependence, by taking into account the filter response curve and the spectrum of the source (using the Keck LRIS optical spectrum of the T4.5 dwarf 2MASS J05591914-1404488 from Burgasser et al. [2003a] as the template for the individual components of 2MASS J1534-2952AB); (3) telescope jitter (0-20 mas of Gaussian jitter); and (4) telescope defocus $( \pm 10 \mu \mathrm{m})$ to account for HST breathing effects. Because the geometrical distortion is location dependent, we used Tiny Tim model PSFs generated for the nearest integer pixel location to the centroid of the binary or single T dwarf. Also, we used the template spectrum closest to the spectral type of the $\mathrm{T}$ dwarf with sufficient wavelength coverage $(0.70-0.96 \mu \mathrm{m})$ from S. K. Leggett's spectral library. ${ }^{12}$

These Tiny Tim model PSFs were used to fit simultaneously for (1) the location of the primary, (2) the location of the secondary, (3) the normalization of the model PSF to the primary, and (4) the flux ratio of the two components. When fitting positions, the supersampled Tiny Tim PSF was interpolated using cubic convolution to the appropriate subpixel location. The best-fit values were found using the amoeba algorithm (e.g., Press et al. 1992) to

12 The optical spectra we used were 2MASS J05591914-1404488 for T4.5 and T5.5 objects (Burgasser et al. 2003a), SDSSp J162414.37+002915.6 for T6 objects (Burgasser et al. 2000), SDSSp J134646.45-003150.4 for T6.5 objects (Burgasser et al. 2000), and Gl 570D for T7.5 objects (Burgasser et al. 2003a). 
TABLE 2

HST OBSERVATIONS

\begin{tabular}{cccrrr}
\hline \hline $\begin{array}{c}\text { Date } \\
\text { (UT) }\end{array}$ & Instrument & Filter & \multicolumn{1}{c}{$\begin{array}{c}\text { Separation } \\
\text { (mas) }\end{array}$} & $\begin{array}{c}\text { Position Angle } \\
\text { (mas) }\end{array}$ & $\Delta$ mag \\
\hline 2000 Aug 18............ & WFPC2 & F814W & $62.8 \pm 1.2$ & $357.1 \pm 0.8$ & $0.30 \pm 0.05$ \\
2006 Jan 19............ & ACS & F814W & $199.0 \pm 1.1$ & $14.5 \pm 0.6$ & $0.28 \pm 0.06$ \\
2006 Apr 11............. & ACS & F814W & $191.2 \pm 1.1$ & $15.5 \pm 0.4$ & $0.30 \pm 0.04$ \\
\hline
\end{tabular}

a The tabulated errors are dominated by the uncertainties in fitting the binary images, which are much larger than the errors in the overall astrometric calibration of WFPC2 and ACS. See $\S 2$ for details.

find the minimum $\chi^{2}$ value of a $1.1^{\prime \prime} \times 1.1^{\prime \prime}$ subimage centered on the binary. The image was cleaned using the IDL routine cr_reject in the Goddard IDL library to identify and mask the numerous cosmic rays in the undithered WFPC2 image pair. Masked pixels were excluded from the computation of the $\chi^{2}$ value. The noise in each pixel was determined from the bias-subtracted raw WFPC2 image, assuming a read noise of $5.3 e^{-}$pixel $^{-1}$ and a gain given by the header keyword ATODGAIN. A grid of PSFs in telescope jitter and defocus were tried, and the fit corresponding to the jitter and defocus combination yielding the lowest $\chi^{2}$ was chosen. For images of 2MASS J1534-2952AB we found that our PSF-fitting routine yielded residuals of $\lesssim 2 \%$ of the peak value in $90 \%$ of pixels with signal-to-noise ratio $\mathrm{S} / \mathrm{N}>3$.

Due to the optical distortion and "34th row" defect present in the WFPC2 (Anderson \& King 1999, 2003), the best-fit pixel locations of each binary component do not exactly correspond to their locations in an undistorted celestial reference frame. To remove these effects, we applied the corrections of Anderson \& King (2003), using a pixel scale of $45.54 \pm 0.01$ mas pixel ${ }^{-1}{ }^{13}$ The 34th row effect could change, for example, the binary separation by as much as 0.7 mas (a systematic shift of $-0.6 \sigma$ ), but because the binary components do not straddle a defective row and are separated by a mere 1.4 pixels, the application of the 34th row and distortion corrections have a negligible effect on the astrometry.

With only two undithered WFPC 2 images of 2MASS J1534$2952 \mathrm{AB}$, it is challenging to quantify the measurement uncertainties, and it is impossible to completely quantify the systematic errors, which arise from an imperfect PSF model and also probably depend on the subpixel positions of the two components given the undersampled nature of the data. Using only the rms scatter of the two measurements, the inferred random errors in separation, P.A., and flux ratio are 0.9 mas, $0.07^{\circ}$, and $0.03 \mathrm{mag}$, respectively. To derive more robust random errors and to investigate the systematic errors, we conducted an extensive Monte Carlo simulation of our fitting routine.

We used WFPC2 F814W images of seven other T dwarfs from the same HST program, all apparently single, to create artificial binaries that we then modeled using our PSF-fitting routine. ${ }^{14}$

\footnotetext{
13 The value of the pixel scale and its uncertainty come from the WFPC2 Instrument Handbook for Cycle 13. This number is consistent with other measurements available in the literature: (1) Holtzman et al. (1995) derived a pixel scale of 45.54 mas pixel $^{-1}$ by comparing commanded telescope offsets in arcseconds to the resulting pixel offsets; (2) Holtzman et al. (1995) also derived a pixel scale of 45.55 mas pixel $^{-1}$ by comparison to an astrometric standard field in M67; (3) Pascu et al. (1998) used the JPL ephemeris of the satellites of Uranus to derive a pixel scale of 45.57 mas pixel $^{-1}$; and (4) Pascu et al. (2004) used the JPL ephemeris of the satellites of Neptune to derive a pixel scale of $45.55 \mathrm{mas} \mathrm{pixel}^{-1}$. The scatter in these pixel scales is consistent with our quoted uncertainty.

${ }_{14}$ These were 2MASS J05591914-1404488 (T4.5), 2MASSI J0937347+ 293142 (T6.0), 2MASSI J1217110-031113 (T7.5), 2MASS J12373919+ 6526148 (T6.5), Gl 570D (T7.5), 2MASSI J1546291-332511 (T5.5), and 2MASSI J2356547-155310 ( T5.5), using spectral types from Burgasser et al. (2006b). The remaining object from this program, 2MASSI J1047538+212423 (T6.5), was unusable for our purposes because it landed on a bad column.
}

Because the WFPC2 PSF is severely undersampled, we only created artificial binaries with integer-shifted positions. It turns out that the WFPC2 locations of the two components of 2MASS J1534-2952AB are at a very nearly integer-shifted separation of 1.4 pixels $(\Delta x \approx 1$ pixel, $\Delta y \approx-1$ pixel). Therefore, in determining the uncertainties and systematic offsets we used only the configuration most nearly matching that of 2MASS J1534-2952AB with a separation of $\sqrt{2}$ pixels and instrumental P.A. of $225^{\circ}$. We found that using any or all of the other three $\sqrt{2}$ configurations gave consistent uncertainties. After subtracting the best-fit model, artificial binary images yielded residual images in which $90 \%$ of pixels with $\mathrm{S} / \mathrm{N}>3$ were below $1 \%-3 \%$ of the peak flux, comparable to the residual images of 2MASS J1534-2952AB.

The images of the single $\mathrm{T}$ dwarfs span a range in $\mathrm{S} / \mathrm{N}$ from about 1.5 mag brighter to 1.3 mag fainter than the primary component of 2MASS J1534-2952AB. We used these images at their native $\mathrm{S} / \mathrm{N}$ when simulating the primary component. To simulate the secondary component, we degraded the $\mathrm{S} / \mathrm{N}$ of the images assuming a flux ratio of $0.30 \mathrm{mag}$. We also tried flux ratios of 0.25 and $0.35 \mathrm{mag}$ to explore the possibility that the uncertainties depend on the assumed flux ratio, but we found that this had an insignificant effect on our predicted uncertainties $\left[<1 \sigma_{\sigma}\right.$, where $\sigma_{\sigma}=\sigma /\left(2 N_{\text {sim }}\right)^{1 / 2}$ and $N_{\text {sim }}$ is the number of simulations]. $\mathrm{S} / \mathrm{N}$ degradation of an image was done by a multiplicative scaling followed by the addition of normally distributed random noise to each pixel, according to the same WFPC2 noise model we used to determine $\chi^{2}$ in the PSF-fitting procedure. In fact, by running simulations where the primary images were degraded to much lower $\mathrm{S} / \mathrm{N}$, we found that all of the single T dwarfs are in a high-S/N regime in which systematic errors (PSF model imperfections) dominate, not random errors (photon noise): our simulations showed no dependence between the $\mathrm{S} / \mathrm{N}$ of the $\mathrm{T}$ dwarf used to construct artificial binaries and the resulting astrometric uncertainties. Therefore, we used the rms of the results from all simulated binaries in order to determine the final uncertainties for 2MASS J1534-2952AB. As expected, the uncertainties in separation, P.A., and flux ratio from our simulations were somewhat larger than those derived from the standard deviation of the measurements from the two 2MASS J1534-2952AB images, since both random and systematic errors have been evaluated in the simulations. In fact, the larger uncertainties are not simply due to averaging over, for example, the many subpixel locations of the single T dwarfs used in the Monte Carlo because the simulated measurements for each single $\mathrm{T}$ dwarf show scatter consistent with the final derived uncertainties.

Table 2 presents our final results for the WFPC2 images, with systematic offsets from the Monte Carlo simulations applied. Our astrometry agrees well (better than $1 \sigma$ ) with the original results of Burgasser et al. (2003b) although our measurement errors are a factor of 8 smaller. Part of this improvement comes from our use of Tiny Tim-computed PSFs, as opposed to the simpler Burgasser et al. (2003b) PSF model of a Gaussian plus empirical residuals. We also used all possible single PSFs in our artificial-binary 
simulations, whereas Burgasser et al. (2003b) used only WFPC2 images of 2MASS J0559-1404, a source that is suspected to be an unresolved binary. ${ }^{15}$ And part of the improved uncertainties is somewhat illusional, as it arises from the different parameter space explored in Monte Carlo simulations by us and Burgaser et al. (2003b). As a check, we ran a suite of simulations more comparable to that of Burgasser et al. (2003b), in which the ranges of artificial binary parameters were 1.0-3.0 pixels in separation, the full range of P.A., and 0.0-1.0 mag in flux ratio. These yielded similar astrometric uncertainties to the published errors, suggesting that the smaller uncertainties we derive are due to our more restricted choice of artificial binary configurations $(\Delta x=1$ pixel, $\Delta y=-1$ pixel) and/or averaging over many single PSFs to reduce systematics associated with any one specific object.

Our improvement to the WFPC2 astrometry was essential in our early attempts to fit the orbit based on Keck data obtained in 2005-2007. However, with the addition of data in 2008, the final $H S T+$ Keck data set has sufficient time baseline and astrometric quality that the choice of WFPC2 astrometry does not highly impact the final orbit fitting results $(\S 3.3 .3)$.

\subsubsection{ACS High Resolution Camera}

The 2MASS J1534-2952AB system was observed on 2006 January 19 and April 11 (UT) with the High Resolution Camera (HRC) of HST ACS by program GO-10559 (PI H. Bouy). The binary is much more widely separated at these epochs than in the WFPC2 observations, but the PSFs of the two components are still blended. We have therefore applied the same Tiny Tim PSFfitting technique described in the previous section to derive the relative astrometry from the ACS images. The primary differences between the WFPC2 and ACS data sets are the following: (1) ACS has much more severe geometric distortion than WFPC2, which changes the shape of the PSF and complicates astrometry because the pixels projected on the sky are not square; and (2) the ACS data are of much lower $\mathrm{S} / \mathrm{N}$, with a total exposure time of only $50 \mathrm{~s}$ for each cosmic-ray rejected, combined dithered image (cf. $1300 \mathrm{~s}$ for a single WFPC2 image). Because of the lower $\mathrm{S} / \mathrm{N}$, we found it unwarranted to fit the ACS images for telescope jitter and defocus as adding these free parameters did not improve the quality of the fits (as verified in the Monte Carlo simulations discussed below). Also, we found that the $\approx 25$ times lower $\mathrm{S} / \mathrm{N}$ of these data almost exactly negates any improvement to the astrometry that might be expected given the larger binary separation at these epochs.

We used distorted model PSFs generated by Tiny Tim to fit for the position and flux of each binary component in images that had been cosmic-ray cleaned (CRSPLIT $=4$ ) by the latest $H S T$ pipeline. Best-fit pixel locations were corrected for geometric distortion using the solution of Anderson \& King (2004; Instrument Science Report 04-15), and we used their measured ACS pixel scale, which was derived by comparing commanded (POSTARG) offsets of HST in arcseconds to the resulting pixel offsets. They derived two such pixel scales for two epochs of observations of

\footnotetext{
15 2MASS J0559-1404 is roughly twice as luminous as objects of similar spectral type (Dahn et al. 2002; Tinney et al. 2003; Vrba et al. 2004). High angular resolution observations have not detected any multiplicity (Burgasser et al. 2003b; M. C. Liu et al. 2008, in preparation), but the source could be a very tight system, e.g., with a 0.5 pixel separation. If so, its multiplicity could confuse any attempts to fit only two single PSFs to the tightest artificial binaries constructed from its image. Interestingly, we found that at the smallest (1.0 pixel) separations, artificial binaries constructed from images of 2MASS J0559-1404 yielded extremely large uncertainties $\left(5\right.$ mas and $15^{\circ}$ ). We did not observe such behavior for any of the other six apparently single T dwarfs, nor were the uncertainties for larger separation binaries made from 2MASS J0559-1404 abnormally large. One natural explanation would be that the source is just marginally resolved in the WFPC2 imaging.
}

47 Tuc, and we adopt the mean and standard deviation of these two: $28.273 \pm 0.006$ mas pixel $^{-1} \cdot{ }^{16}$ For each epoch, we adopted the mean of the measurements from all four dithered images for the binary parameters of 2MASS J1534-2952AB (Table 2).

Again, to investigate the measurement errors thoroughly, we performed Monte Carlo simulations of our fitting routine. We used images of single brown dwarfs to construct artificial binaries in configurations resembling 2MASS J1534-2952AB. At both ACS epochs, the binary is well represented by integer-pixel shifts on a grid where $\Delta x=(6,7)$ and $\Delta y=(-3,-2,-1,0,1)$. There have been no HST ACS science programs dedicated to studying single brown dwarfs; however, ACS images of the single brown dwarfs 2MASS J00361617+1821104 (L3.5; Kirkpatrick et al. 2000) and 2MASS J05591914-1404488 (T4.5; Burgasser et al. 2006b) were obtained for calibration purposes and are available in the HST Archive (CAL/ACS-10374, PI Giavalisco). We found that despite the large difference in spectral types, any corresponding difference in the PSF does not alter the results of the Monte Carlo simulations. The $\mathrm{S} / \mathrm{N}$ of each of these single objects is much higher than that of 2MASS J1534-2952AB, so we degraded the $\mathrm{S} / \mathrm{N}$ of the single brown dwarfs for the artificial binary simulations. In fact, by varying the $\mathrm{S} / \mathrm{N}$ of the simulations, we found that the ACS data for 2MASS J1534-2952AB are well in the S/N regime dominated by random photon noise, while the images of the single objects are in a high-S/N regime dominated by systematic errors (akin to the WFPC2 images of 2MASS J1534-2952AB). Therefore, given that we have four images, we divide the rms of the Monte Carlo results by $\sqrt{4}$ to represent the final uncertainties.

Table 2 contains our final ACS results. ${ }^{17}$ Note that the ACS data are contemporaneous with our Keck LGS data, and observations by the two telescopes in 2006 separated by less than 1 month show excellent agreement. However, the relatively low $\mathrm{S} / \mathrm{N}$ of the ACS data means that it has the larger astrometric errors. Our simulations confirm that if the ACS data were of higher $\mathrm{S} / \mathrm{N}$ (i.e., longer exposure times than $50 \mathrm{~s}$ ), the resulting astrometric precision would be much better than, instead of comparable to, the WFPC2 astrometric uncertainties.

\subsection{IRTF/SpeX Spectroscopy}

We obtained low-resolution $(R \approx 150)$ integrated-light spectra of 2MASS J1534-2952AB on 2008 May 16 UT from NASA's Infrared Telescope Facility (IRTF) located on Mauna Kea, Hawaii. Conditions were photometric with seeing of about $0.7^{\prime \prime}$ FWHM near the target. We used the facility near-IR spectrograph Spex (Rayner et al. 1998) in prism mode, obtaining 0.8-2.5 $\mu \mathrm{m}$ spectra in a single order. We used the $0.5^{\prime \prime}$ wide slit, oriented at the parallactic angle to minimize the effect of atmospheric dispersion. 2MASS J1534-2952AB was nodded along the slit in an ABBA pattern, with individual exposure times of $180 \mathrm{~s}$, and observed over an air-mass range of $1.64-1.60$ as it rose. The telescope was guided during the exposures using images obtained with the near-IR slit-viewing camera. The total on-source exposure time was $720 \mathrm{~s}$. We observed the A0 V star HD 142851 contemporaneously for flux and telluric calibration. All spectra were reduced using version 3.4 of the SpeXtool software package (Vacca et al.

16 Comparison of WFPC2 PC images of 47 Tuc (GO-8267, PI Gilliland) with ACS WFC images (GO-10775, PI A. Sarajedini) shows excellent agreement between our adopted pixel scales for the two instruments, at the level of $2 \times 10^{-4}$, which is well below the other errors in the measurements (J. Anderson 2007, private communication).

17 After our paper was submitted, Bouy et al. (2008) reported an analysis of the same ACS images. Their results agree with ours to within the stated uncertainties. Their errors are slightly smaller than ours in separation ( 0.9 mas compared to 1.1 mas) and much smaller in P.A. $\left(0.1^{\circ}\right.$ compared to $\left.0.5^{\circ}\right)$. 


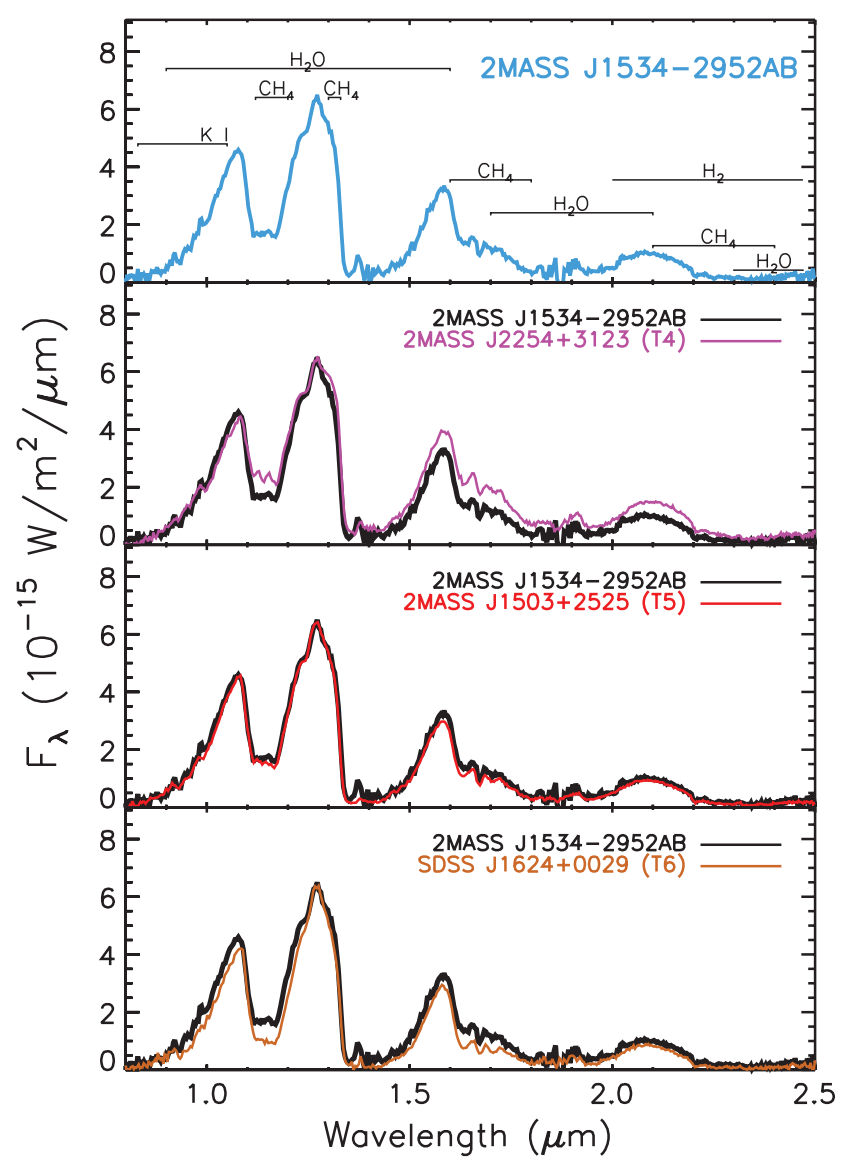

FIG. 2.-Top: Near-IR spectrum of 2MASS J1534-2952AB obtained with the IRTF/SpeX spectrograph. Other panels: Same spectrum of 2MASS J1534-2952AB plotted as a thick black line. Spectra of T dwarf spectral standards from Burgasser et al. (2006b) are plotted as colored lines. The spectra have been normalized by their peak flux.

2003; Cushing et al. 2004). The reduced IRTF/Spex spectrum is plotted in Figure 2 and compared to T dwarf spectral standards from Burgasser et al. (2006b). Visual examination shows an excellent match to the T5 spectral standard 2MASS J1503+2525, as does measurement of the Burgasser et al. (2006b) spectral indices for 2MASS J1534-2952AB: $\mathrm{H}_{2} \mathrm{O}-J=0.271$ ( T4.8), $\mathrm{CH}_{4}-J=0.420$ (T4.8), $\mathrm{H}_{2} \mathrm{O}-H=0.345$ (T5.0), $\mathrm{CH}_{4}-H=$ 0.430 (T5.0), and $\mathrm{CH}_{4}-K=0.224$ (T5.1), with spectral type estimates based on the polynomial fits to the indices from Burgasser (2007b).

\section{RESULTS}

\subsection{Resolved Photometry and Spectral Types}

We use our measured flux ratios and the published JHK photometry from Knapp et al. (2004) to derive resolved IR colors and magnitudes for 2MASS J1534-2952AB on the MKO system. We use the HST photometry from Burgasser et al. (2003b) in determining the resolved $\mathrm{F} 814 \mathrm{~W}$ magnitudes. Then to infer spectral types for the individual components, we compare these to magnitudes and colors of ultracool dwarfs from Knapp et al. (2004) and Chiu et al. (2006) excluding known binaries. We use near-IR spectral classifications from the Burgasser et al. (2006b) scheme. We assume that the components of 2MASS J1534-2952AB are themselves single, not unresolved binaries.

Figure 3 shows that component A has IR colors most typical of T4.5-T5 dwarfs, and component B is most similar to T5-T6

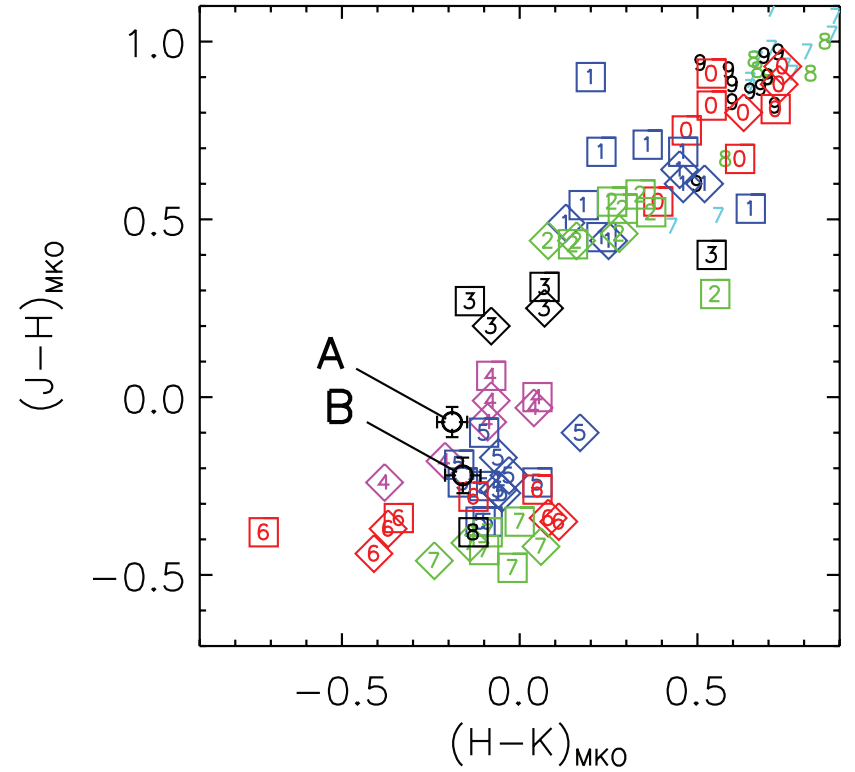

FIG. 3.-Near-IR colors of 2MASS J1534-2952AB compared with nearby single late L and T dwarfs from Knapp et al. (2004) and Chiu et al. (2006) and individual components of resolved binaries from McCaughrean et al. (2004), Burgasser et al. (2005b, 2006c), Liu \& Leggett (2005), and Liu et al. (2006). The photometry errors are comparable to or smaller than the size of the plotting symbols. The numbers indicate the near-IR spectral subclass of the objects, with half subclasses being rounded down (e.g., T3.5 is labeled as " 3 "), and objects of the same subclass plotted in the same color. The late L dwarfs (classified on the Geballe et al. [2002] scheme) are plotted as bare numbers. The T dwarfs (on the Burgasser et al. [2006b] scheme) are plotted as circumscribed numbers, with squares for integer subclasses (e.g., T3) and diamonds for half subclasses (e.g., T3.5).

dwarfs. The individual absolute IR magnitudes (given in Table 3) give similar results. The "faint" polynomial fits for absolute magnitude as a function of spectral type from Liu et al. (2006) give $M(J)=\{14.5,14.6,14.7,14.9,15.2\} \mathrm{mag}, M(H)=\{14.6$, $14.8,15.0,15.2,15.5\} \mathrm{mag}$, and $M(K)=\{14.7,14.9,15.1$, $15.4,15.7\}$ mag for near-IR spectral types of T4.5, T5, T5.5, T6, and T6.5, respectively. Averaging the same data for each individual subclass gives $M(J)=\{13.9 \pm 0.6,14.1,14.4 \pm 0.4$, $15.0 \pm 0.5,15.1 \pm 0.5\} \mathrm{mag}, M(H)=\{14.0 \pm 0.6,14.2,14.6 \pm$ $0.4,15.3 \pm 0.4,15.4 \pm 0.5\} \mathrm{mag}$, and $M(K)=\{14.0 \pm 0.5$, $14.3,14.6 \pm 0.4,15.5 \pm 0.7,15.6 \pm 0.9\} \mathrm{mag}$, where the uncertainties are the rms of the photometry for each subclass (and no listed uncertainties for subclasses with only one object). Altogether, the absolute magnitudes suggest types of T5-T5.5 for component A and T5.5-T6 for component B.

The resolved $\left(\mathrm{CH}_{4} \mathrm{~S}-\mathrm{H}\right)$ colors provide a third means to estimate the spectral types, as these track the $H$-band methane absorption, which correlates well with overall near-IR spectral type (e.g., Fig. 2 of Tinney et al. 2005). First, we compute individual $\mathrm{CH}_{4} S$ magnitudes for 2MASS J1534-2952AB using the $\mathrm{CH}_{4} S$ flux ratio from our LGS images, the integrated-light photometry of $H=14.74 \pm 0.03 \mathrm{mag}$ from Knapp et al. (2004), and an integrated-light color of $\left(\mathrm{CH}_{4} s-H\right)=-0.33$ mag synthesized from the near-IR spectrum of Burgasser et al. (2006b). Including the measurement errors in the flux ratios and $H$-band photometry, we find $\mathrm{CH}_{4} S=15.06 \pm 0.04$ and $15.27 \pm 0.04 \mathrm{mag}$ and $\left(\mathrm{CH}_{4} S-H\right)=-0.30 \pm 0.05$ and $-0.37 \pm 0.05$ mag for components A and B, respectively. Note that the relative $\left(\mathrm{CH}_{4} S-H\right)$ color of the two components is known to higher precision, since the above computed colors for the two components contain the same 0.03 mag error that originates from the integrated-light $H$-band photometry. (In other words, the 0.05 mag uncertainties 
TABLE 3

Resolved Properties of 2MASS J1534-2952AB

\begin{tabular}{|c|c|c|}
\hline Property & 2MASS J1534-2952A & 2MASS J1534-2952B \\
\hline $\mathrm{F} 814 \mathrm{~W}-J(\mathrm{mag})$ & $4.95 \pm 0.04$ & $5.10 \pm 0.04$ \\
\hline$J-H(\mathrm{mag})$ & $-0.08 \pm 0.04$ & $-0.21 \pm 0.04$ \\
\hline $\mathrm{CH}_{4} s-H(\mathrm{mag})^{\mathrm{a}}$. & $-0.30 \pm 0.05$ & $-0.37 \pm 0.05$ \\
\hline$H-K(\mathrm{mag}) \ldots \ldots \ldots$ & $-0.17 \pm 0.04$ & $-0.17 \pm 0.04$ \\
\hline$J-K(\mathrm{mag}) \ldots \ldots \ldots \ldots \ldots \ldots \ldots \ldots \ldots \ldots \ldots \ldots \ldots \ldots$ & $-0.25 \pm 0.04$ & $-0.38 \pm 0.04$ \\
\hline 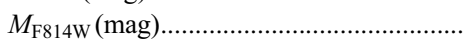 & $19.57 \pm 0.04$ & $19.87 \pm 0.05$ \\
\hline$M_{J}(\mathrm{mag})$ & $14.61 \pm 0.05$ & $14.77 \pm 0.05$ \\
\hline$M_{H}(\mathrm{mag})$ & $14.69 \pm 0.05$ & $14.98 \pm 0.05$ \\
\hline$M_{K}(\mathrm{mag})$ & $14.86+0.05$ & $15.15+0.05$ \\
\hline Estimated spectral type $\mathrm{b}^{\mathrm{b}}$. & $\mathrm{T} 5.0 \pm 0.5$ & $\mathrm{~T} 5.5 \pm 0.5$ \\
\hline $\log \left(L_{\mathrm{bol}} / L_{\odot}\right)^{\mathrm{a}}$ & $-5.015 \pm 0.019$ & $-5.093 \pm 0.019$ \\
\hline
\end{tabular}

NoTE.-All infrared photometry on the MKO photometric system.

${ }^{\mathrm{a}}$ The difference in the $\left(\mathrm{CH}_{4} \mathrm{~s}-\mathrm{H}\right)$ color of the two components is $0.07 \pm 0.02 \mathrm{mag}$, i.e., better constrained than the quadrature sum of the measurement errors tabulated here (see $\S 3.1$ ). Similarly, the difference in $\log \left(L_{\mathrm{bol}} / L_{\odot}\right)$ is $0.078 \pm 0.016 \mathrm{dex}$, since this quantity is independent of the distance uncertainty.

${ }^{\mathrm{b}}$ Based on the Burgasser et al. (2006b) near-IR classification scheme.

in the colors of the two components are not independent, but correlated.) Removing this effect gives a relative color of $\Delta\left(\mathrm{CH}_{4} s-H\right)=0.07 \pm 0.02 \mathrm{mag}$ between $\mathrm{A}$ and B; i.e., greater methane absorption in component $\mathrm{B}$ is detected.

To determine the behavior of $\left(\mathrm{CH}_{4} S-H\right)$ with near-IR spectral type, we synthesized colors from the Spex Prism Spectral Library collection, which contains low-resolution spectra of $68 \mathrm{~T}$ dwarfs after removing spectrally peculiar objects and known binaries. ${ }^{18}$ Figure 4 plots the results. We fit a second-order polynomial for the dependence of color on near-IR spectral type and vice versa:

$\left(\mathrm{CH}_{4} s-H\right)=-0.400+0.07718 \times \mathrm{SpT}-0.0029736 \times \mathrm{SpT}^{2}$,

$$
\mathrm{SpT}=19.40-19.698\left(\mathrm{CH}_{4} s-H\right)-8.3600\left(\mathrm{CH}_{4} s-H\right)^{2} \text {, }
$$

where $\mathrm{SpT}=20$ for T0, 21 for T1, etc. The rms scatter about the fits is $0.02 \mathrm{mag}$ and 0.3 subclasses, respectively. Using these polynomial relations, the observed $\left(\mathrm{CH}_{4} \mathrm{~S}-\mathrm{H}\right)$ colors give spectral types of T4.5 \pm 0.7 and T5. $6 \pm 0.6$ for components A and $\mathrm{B}$, respectively, where the spectral type uncertainties come from formal propagation of the uncertainty in the colors. In addition, just as the relative $\left(\mathrm{CH}_{4} S-H\right)$ colors of the two components are known more accurately than the absolute colors, we compute a relative spectral type of $1.1 \pm 0.3$ subclasses between components $\mathrm{A}$ and $\mathrm{B}$.

Combining the inferences from the $J H K$ colors, the absolute magnitudes, and the $\left(\mathrm{CH}_{4} \mathrm{~s}-\mathrm{H}\right)$ colors, we adopt spectral type estimates of $\mathrm{T} 5 \pm 0.5$ and $\mathrm{T} 5.5 \pm 0.5$ for the two components. The relative $\left(\mathrm{CH}_{s}-H\right)$ color favors a slightly larger spectral type difference than the absolute magnitudes but consistent with the adopted uncertainty. (Also the $T_{\text {eff }}$ difference of the two components computed in $\S 4.3$ favors a 0.5 subclass difference.)

Higher order multiple systems are very rare among ultracool binaries, with an estimated frequency of $3_{-1}^{+4} \%$ (Burgasser et al. 2007), and thus a priori we do not expect 2MASS J1534$2952 \mathrm{AB}$ to fall into this category. The colors and magnitudes are

\footnotetext{
${ }^{18}$ See http://www.browndwarfs.org/spexprism. The T dwarf spectra are compiled from Burgasser et al. (2004, 2006a, 2006b, 2007a), Chiu et al. (2006), Looper et al. (2007), and Liebert \& Burgasser (2007).
}

consistent with the system being composed of only two components, and not being a partially resolved higher order multiple system. If component B was actually an equal-mass binary, the absolute magnitudes of its components would be $0.75 \mathrm{mag}$ fainter than the integrated light of $\mathrm{B}$, meaning $M(J, H, K)=\{15.5$, $15.9,16.0\}$ mag. This would suggest a spectral type around T7, based on the polynomial relations in Liu et al. (2006), which is clearly too late type compared to the integrated-light spectrum and the observed near-IR colors of B.

\subsection{Bolometric Luminosities}

To measure the $L_{\mathrm{bol}}$ for the system, we combine our SpeX $0.9-2.4 \mu \mathrm{m}$ spectrum with the published integrated-light F814W, $L^{\prime}$-band, and Spitzer IRAC thermal-IR photometry and uncertainties (Burgasser et al. 2003b; Knapp et al. 2004; Golimowski et al.

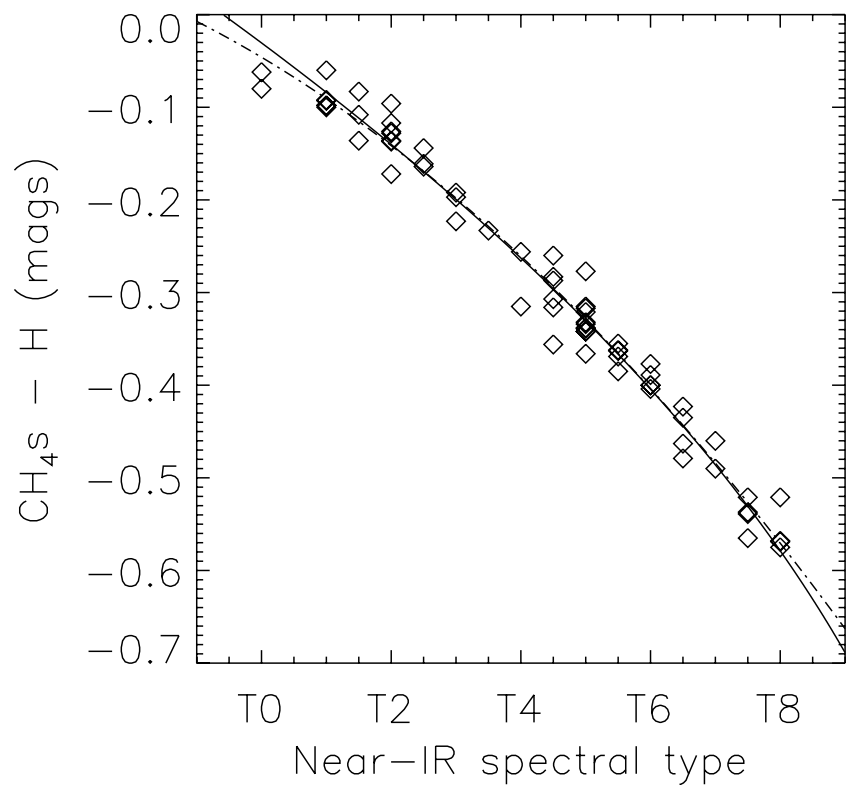

FIG. 4.- $\left(\mathrm{CH}_{4} s-H\right)$ color vs. near-IR spectral type for T dwarfs. The plotting symbols are the synthesized colors from published low-resolution near-IR spectra of T dwarfs, excluding objects that are spectrally peculiar and known binaries (see $\S 3.1$ for references). The lines represent second-order polynomial fits to the data, with the solid line being the spectral type as a function of color (i.e., the inverse fit) and the dotted line being the color as a function of spectral type. 
2004a; Patten et al. 2006). ${ }^{19}$ We flux-calibrated the SpeX data using the published $H$-band MKO photometry from Knapp et al. (2004). For the Spitzer IRAC data, we adopted the photometric calibration and an overall $2 \%$ absolute uncertainty based on Reach et al. (2005). We extended the binary's spectral energy distribution (SED) to short wavelengths by linearly interpolating from the F814W datum to zero flux at zero wavelength and to long wavelengths by assuming a Rayleigh-Jeans spectrum beyond the reddest Spitzer bandpass $(7.87 \mu \mathrm{m})$; this extrapolation increases the total flux by $2 \%$.

We then integrated the SED, using a Monte Carlo approach to account for all the measurement errors. We find $\log \left(L_{\mathrm{bol}} / L_{\odot}\right)=$ $-4.751 \pm 0.011 \mathrm{dex}$ for the system, with the uncertainty increasing to $0.018 \mathrm{dex}$ after including the uncertainty in the distance. (As discussed in $\S 4$, we keep track of these independent uncertainties in our calculations.) The largest uncertainty in the integration arises from the 0.03 mag uncertainty in the integrated-light photometry used to normalize the SpeX spectrum. We cross-checked our method using the same data for the T4.5 dwarf 2MASS J0559-1404 and found excellent agreement with the $L_{\text {bol }}$ measured by Cushing et al. (2006) using absolutely flux-calibrated spectra from 0.6 to $15 \mu \mathrm{m}$.

The computed total $L_{\text {bol }}$ agrees well with that inferred from using the $K$-band bolometric corrections $\left(\mathrm{BC}_{K}\right)$ from Golimowski et al. (2004a), namely, using the resolved $K$-band absolute magnitudes and the estimated spectral types, which would give $\log \left(L_{\mathrm{bol}} / L_{\odot}\right)=-4.97 \pm 0.06$ and $-5.06 \pm 0.06 \mathrm{dex}$ for the individual components and thus $\log \left(L_{\mathrm{bol}} / L_{\odot}\right)=-4.71 \pm 0.08 \mathrm{dex}$ for the total system. However, the uncertainties are larger when using $\mathrm{BC}_{K}$ to derive $L_{\mathrm{bol}}$, since this incorporates the uncertainties arising from the 0.5 subclass uncertainty $(0.06$ mag in bolometric magnitude) and the intrinsic scatter in the Golimowski et al. (2004a) $\mathrm{BC}_{K}$ relation $(0.13 \mathrm{mag})$. In short, direct integration of the observed SED is more accurate.

To apportion the observed total $L_{\text {bol }}$ into the individual components, we assume that the observed $K$-band flux ratio of the system represents the luminosity ratio. This would be exactly correct if the two components had identical spectral types (and neglecting photometric variability). To account for the difference in spectral types, we generate a Monte Carlo distribution of $\mathrm{BC}_{K}$ values for each component using the Golimowski et al. (2004a) polynomial fit as a function of spectral type subject to the following rules: the spectral type of component $\mathrm{A}$ is uniformly distributed from T4.5 to T5.5, the spectral type of component B is no later than $\mathrm{T} 6$, and the difference in their spectral types is at least $0.5 \mathrm{sub}-$ classes. This produces an average difference in $\mathrm{BC}_{K}$ between the two components of $0.09 \mathrm{mag}$ and an rms of $0.03 \mathrm{mag}$. Thus, we find $\log \left(L_{\mathrm{bol}} / L_{\odot}\right)=-5.015 \pm 0.019$ and $-5.093 \pm 0.019 \mathrm{dex}$ for the two components, including the uncertainty in the distance.

\subsection{Dynamical Mass Determination}

\subsubsection{Orbit Fitting Using Markov Chain Monte Carlo}

We have data at nine independent epochs, which is formally sufficient to determine the seven parameters of a visual binary orbit

\footnotetext{
19 The $L^{\prime}$-band photometry for this system appears to be anomalous, as Leggett et al. (2007b) show that the $\left([3.5]-L^{\prime}\right)$ color is $\sim 0.2$ mag redder than any other T dwarf and $\sim 0.3$ mag redder than objects of similar spectral type. New $L^{\prime}$-band photometry by S. Leggett et al. (2008, private communication) from 2008 July using UKIRT/UIST finds an integrated flux of $L^{\prime}=12.99 \pm 0.02 \mathrm{mag}$. This is somewhat fainter than the value from Golimowski et al. (2004a), which was obtained while thermal photometry with UIST was still being commissioned (S. Leggett 2008, private communication). The new measurement makes the [3.55] $-L^{\prime}$ color of the system typical for its spectral type. Either way, this issue has a negligible effect on the computed $L_{\mathrm{bol}}$.
}

given our measurements (nine positions plus nine times). However, two pairs of measurements are separated by only 1 month (2006 April/May and 2007 March/April), and the cadence of the orbital phase covered is limited, with the HST WFPC2 datum being taken almost $5 \mathrm{yr}$ before the next epoch. While standard gradient-descent (Levenberg-Marquardt) least-squares techniques would be sufficient to derive an orbit (§ 3.3.2), we also would like to accurately determine the probability distribution of the orbit parameters (which may not be normally distributed) and the associated degeneracies. For epochs with Keck data taken in multiple filters, we choose the filter with the smallest astrometry errors.

Thus, we first used a combination of gradient-descent techniques from random starting points and simulated-annealing techniques to isolate the class of potential orbital solutions near a reduced $\chi^{2}\left(\tilde{\chi}^{2}\right)$ of 1 . Then, to fully explore this class of solutions, we used a Markov chain Monte Carlo (MCMC) approach (e.g., Bremaud 1999). MCMC provides a means to explore the multidimensional parameter space inherent in fitting visual orbits that is computationally efficient, is able to discern the degeneracies and nonGaussian uncertainties in the fit, and allows for incorporation of a priori knowledge. In short, the MCMC approach is distinct from ordinary Monte Carlo methods in that instead of completely random steps through the model parameter space, the steps are chosen such that the resulting number of samples (the "chain") is asymptotically equivalent to the posterior probability distribution of the parameters being sought (for explications of applying MCMC to astronomical data see Tegmark et al. 2004; Ford 2005; Gregory 2005).

We parameterized the binary's orbit using the standard seven parameters: period $(P)$, semimajor axis $(a)$, inclination $(i)$, epoch of periastron $\left(T_{0}\right)$, P.A. of the ascending node $(\Omega),{ }^{20}$ eccentricity $(e)$, and the argument of periastron $(\omega)$. We used an MCMC chain length of $2 \times 10^{8}$, with the parameters stored every hundredth iteration. We started the chain at the global minimum found by the simulated-annealing and gradient-descent algorithms. We used the Metropolis-Hastings algorithm to sample the joint probability distribution with a variant of the usual Gibbs sampler. Instead of choosing one of the seven parameters to increment or decrement, we chose instead to move randomly either forward or backward along one of seven orthogonal directions in the parameter space. These directions were initially chosen to be along the individual coordinate axes of our seven parameters. Then, every $5 \times 10^{5}$ iterations, the covariance matrix of the seven parameters was calculated and new directions chosen along the directions corresponding to the eigenvectors of the covariance matrix. After covariance matrix calculation, the trial step size was set to be the square root of the covariance matrix eigenvalues. This enabled the long, thin, curved minima in our parameter space to be sampled much more efficiently. Each set of $5 \times 10^{5}$ iterations can thus be thought of as its own subchain with fixed directions. As the first $5 \times 10^{5}$ iterations use a less efficient sampling (sampling directions aligned with the seven parameters themselves), we treated this part of the chain as the "burn-in" time, neglecting it in the final analysis. As the chain was running, the trial step size in each direction was continuously scaled over a timescale of 700 steps so that the success rate of jumps averaged to 0.25 . The correlation length of our most correlated chain, as defined in Tegmark et al. (2004), was $2 \times 10^{4}$ for the orbital period $(P)$, with equal or smaller correlation lengths for other parameters. This gives an effective length of the chain of $\sim 1 \times 10^{4}$, which in turn gives statistical uncertainties in the parameter errors of about $1 /\left(10^{4}\right)^{1 / 2}=$ $1 \%$, i.e., negligible. These uncertainties in the errors are consistent with the results from running multiple test chains.

\footnotetext{
20 For visual binaries, there is a $180^{\circ}$ ambiguity in determining $\Omega$, which can only be resolved with radial velocity information.
} 

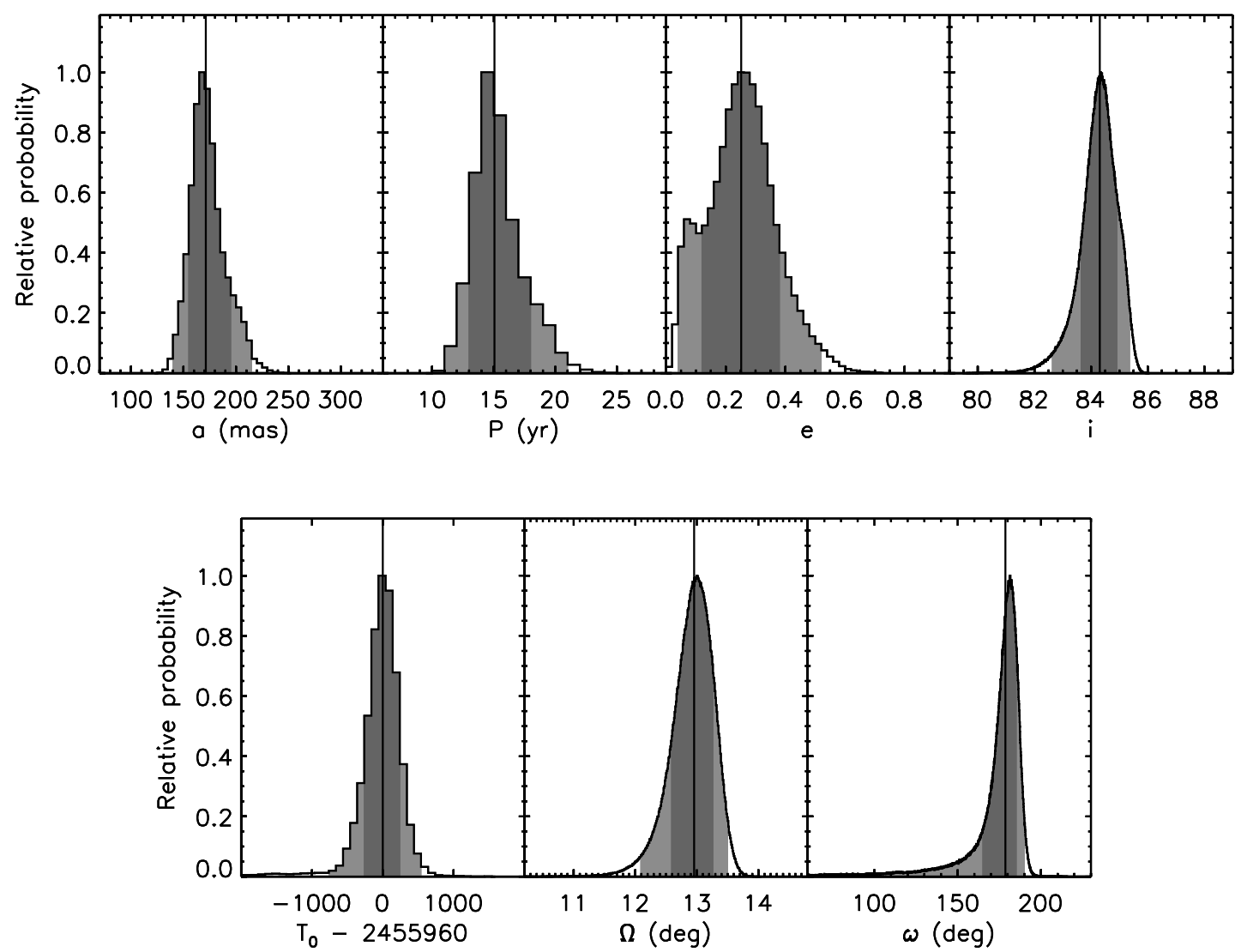

FIG. 5.-Resulting MCMC probability distributions of orbital parameters: semimajor axis $(a)$, orbital period $(P)$, eccentricity $(e)$, inclination $(i)$, epoch of periastron

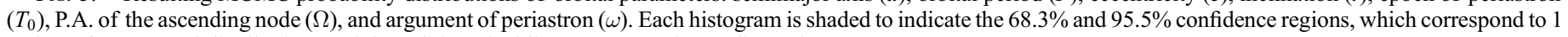
and $2 \sigma$ for a normal distribution, and the solid vertical line represents the median value.

By making steps of the same size in the positive and negative directions for these parameters in constructing the Markov chain, we would implicitly assume that our prior knowledge of these parameters is a uniform distribution. This is not an accurate representation of our prior knowledge since, for example, binaries with periods between $10^{2}$ and $10^{3} \mathrm{yr}$ are not 10 times more common than binaries with periods between 10 and $10^{2} \mathrm{yr}$. We therefore applied a prior to the likelihood function in the MCMC fitting where $P$ and $a$ are distributed evenly in logarithm and where the parameters $e \cos \omega$ and $e \sin \omega$ are uniformly distributed, rather than $e$ and $\omega$, to save the algorithm from unnaturally preferring circular solutions. This is equivalent to the $f(e)=2 e$ distribution as discussed by, e.g., Duquennoy \& Mayor (1991). The very small effect of the choice of prior is discussed below. ${ }^{21}$

\footnotetext{
${ }^{21}$ Some care is also needed in handling the astrometric calibration errors of the three instruments used in our analysis. In practice, most of the constraints come from the HST WFPC2 discovery epoch from 2000 August and the six Keck LGS epochs from 2005 to 2008 . Given the large time difference, the two data sets essentially constrain different portions of the parameter space. Some extra information is provided by the HST ACS data but to a much lesser degree since it is contemporaneous with the Keck LGS data and has 2-4 times larger errors. (In fact, the MCMC fitting gives basically the same results if the ACS data are excluded.) Thus, simply applying the NIRC2 astrometric calibration error of $0.1 \%$ in pixel scale and $0.07^{\circ}$ in P.A. to all six Keck epochs would incorrectly treat this as a random error, when in fact the uncertainty in the NIRC2 calibration globally impacts the overall solution of, e.g., the semimajor axis and the P.A. of the line of nodes. Therefore, we do the following: (1) we apply the errors in the NIRC2 calibration in quadrature to the HST ACS and HST WFPC2 measurements before orbit fitting, and (2) once the semimajor axis distribution has been determined, we apply the uncertainty in the NIRC2 pixel scale in determining the error on the total mass. The net effect is negligible, given the much larger ACS errors compared to the Keck LGS data and the fact that the error in the total mass is dominated by the parallax error (which is 15 times larger than the NIRC2 pixel scale uncertainty).
}

As a consistency check, we also ran our MCMC fitting code on the astrometric data for the binary L dwarf 2MASSW J0746+ 2000AB from Bouy et al. (2004). We found excellent agreement between the orbital parameters derived by us (using MCMC) and by Bouy et al. (2004) (using a variety of $\chi^{2}$ minimization approaches). Not only do the results agree to within the quoted errors, but there is better than $\approx 1 \%$ agreement on the best-fit results and better than $\approx 20 \%$ agreement on the $95 \%$ confidence intervals.

\subsubsection{Fitting Results}

Figure 5 shows the resulting probability distributions for the orbital parameters from the MCMC chain. The probability distributions are clearly not Gaussian. For a given parameter, we adopt the median as the result and describe a confidence limit of $X \%$ as simply the $\frac{1}{2} \pm X / 200$ bounds of the sorted sample. At $68 \%$ (95\%) confidence, we find a modest eccentricity of $0.25_{-0.13(0.20)}^{+0.11(0.25)}$, an orbital period of $15.1_{-1.6(3.1)}^{+2.3(5.1)} \mathrm{yr}$, and a semimajor axis of $171_{-13(27)}^{+19(1)}$ mas $\left(2.3_{-0.2(0.4)}^{+0.3(0.6)}\right.$ AU including the uncertainties in the plate scale and parallax). Two of the orbital angles are very well constrained, the inclination $i=84.3_{-0.6(1.7)}^{+0.6(1.0)} \mathrm{deg}$ (nearly edgeon) and the P.A. of the ascending node $\Omega=13.0_{-0.3(0.9)}^{+0.3(0.5)} \mathrm{deg}$. The final results are summarized in Table 4.

Figure 6 shows the strong correlation between the determination of the orbital period and the eccentricity. It illustrates that there are two classes of possible orbits: one branch having shorter periods and smaller semimajor axes and the other branch having longer periods and larger semimajor axes. Figure 7 shows that the short- $P$ branch orbits have just passed apoastron $\left(\omega \approx 179^{\circ}\right.$, $\Omega \approx 13^{\circ}$ ). This is the favored solution, with $98 \%$ of the steps in the MCMC chain residing in this branch (using $e=0$ as the dividing criterion in the $P$-e plane). However, a nearly circular 
TABLE 4

Derived Orbital Parameters for 2MASS J1534-2952AB

\begin{tabular}{|c|c|c|c|c|}
\hline \multirow[b]{2}{*}{ Parameter } & \multicolumn{3}{|c|}{ MCMC } & \multirow[b]{2}{*}{ ORBIT } \\
\hline & Median & $68.3 \%$ c.l. & $95.5 \%$ c.l. & \\
\hline Time of periastron $T_{0}-2,400,000.5$ (MJD) .................... & $55960^{\mathrm{a}}$ & $-240,210$ & $-740,450$ & $56024 \pm 347$ \\
\hline Orbital period $P(\mathrm{yr})$ & 15.1 & $-1.6,2.3$ & $-3.1,5.1$ & $15.2 \pm 2.6$ \\
\hline Semimajor axis $a$ (mas) & 171 & $-13,19$ & $-27,41$ & $172 \pm 22$ \\
\hline Semimajor axis ${ }^{\mathrm{b}} a(\mathrm{AU})$ & 2.3 & $-0.2,0.3$ & $-0.4,0.6$ & $2.3 \pm 0.3$ \\
\hline Inclination $i(\mathrm{deg})$ & 84.3 & $-0.6,0.6$ & $-1.7,1.0$ & $84.3 \pm 0.8$ \\
\hline Eccentricity $e$ & 0.25 & $-0.13,0.11$ & $-0.20,0.25$ & $0.24 \pm 0.16$ \\
\hline 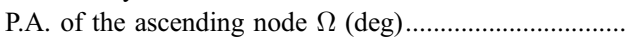 & 13.0 & $-0.3,0.3$ & $-0.9,0.5$ & $13.0 \pm 0.4$ \\
\hline 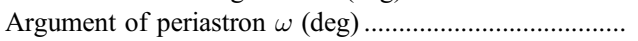 & 179 & $-14,6$ & $-83,11$ & $178 \pm 10$ \\
\hline Fitted total mass $\left(M_{\odot}\right)$ & 0.0556 & $-0.0017,0.0019$ & $-0.004,0.004$ & $0.056 \pm 0.004$ \\
\hline Final total mass $\left(M_{\odot}\right)$ & 0.056 & $-0.003,0.003$ & $-0.006,0.007$ & $0.056 \pm 0.005$ \\
\hline
\end{tabular}

Notes.- Median values and confidence limits for orbital parameters derived from our default MCMC fitting, which uses a prior distribution flat in $\log P$ and $\log a$. The "fitted total mass" represents the direct MCMC results from fitting the observed orbital motion of the two components. The "final total mass" includes the additional $4.9 \%$ error from the uncertainties in the parallax and the Keck /NIRC2 pixel scale; the former is $\approx 15$ times larger than the latter. The final mass distribution is essentially Gaussian. The rightmost column gives the results from the ORBIT routine by Forveille et al. (1999) (see $\S 3.3 .2)$.

a 2012 February 3 UT.

${ }^{\mathrm{b}}$ Includes the uncertainty in the parallax and pixel scale.

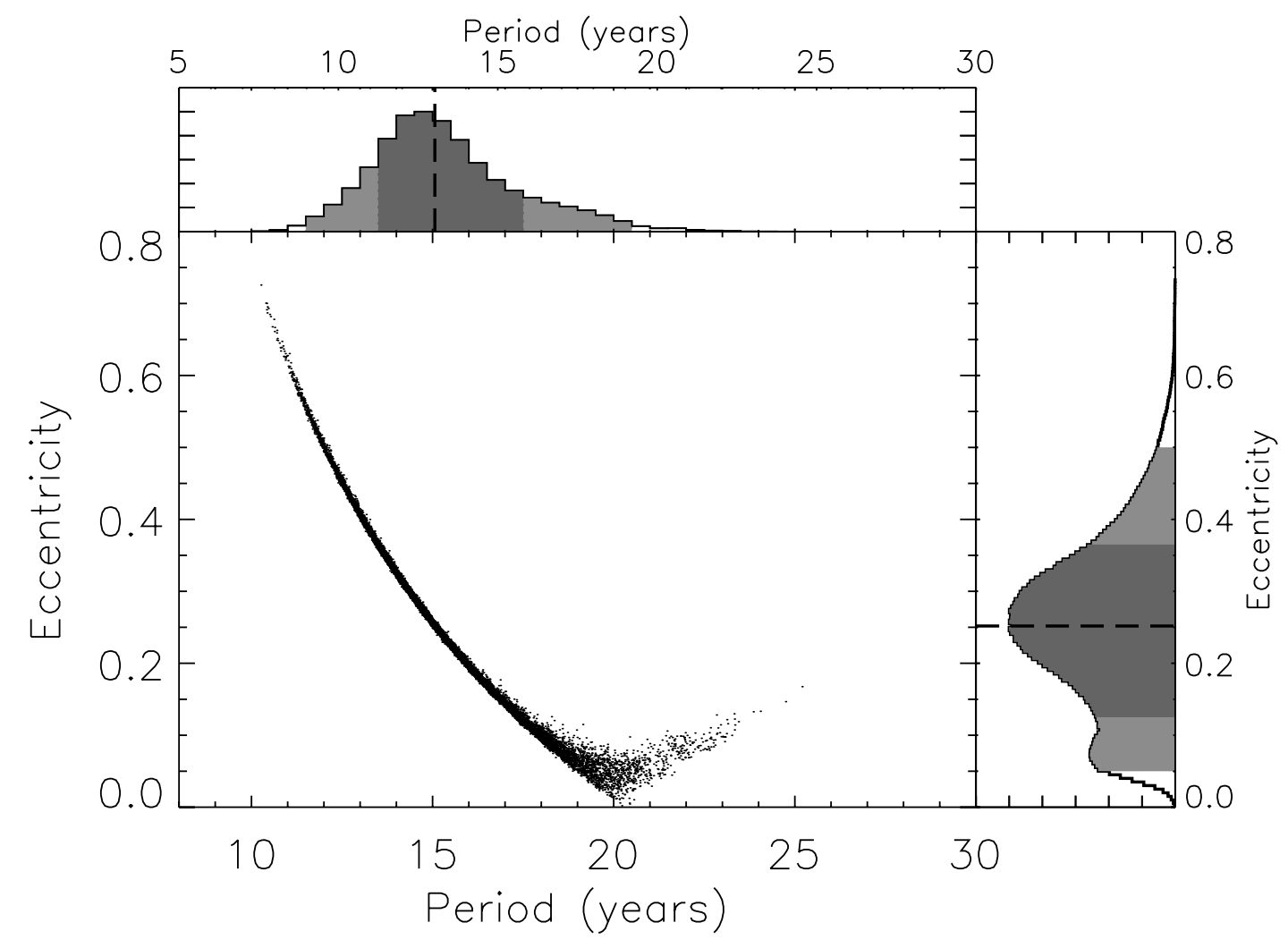

FIG. 6.- Results from MCMC determination of the orbital period and eccentricity for 2MASS J1534-2952AB, illustrating the degeneracy between the two parameters. The central plot shows all the values in the MCMC chain. Two branches of possible orbits are seen, a short-period $(P<20$ yr) branch and a long-period $(P>20 \mathrm{yr})$ one. About $98 \%$ of the MCMC chain steps are in the short-period branch. The top and side plots show the resulting probability distributions of $P$ and $e$. Each histogram is shaded to indicate the $68.3 \%$ and $95.5 \%$ confidence limits, which correspond to 1 and $2 \sigma$ for a normal distribution, and the dashed vertical lines represent the median values. 


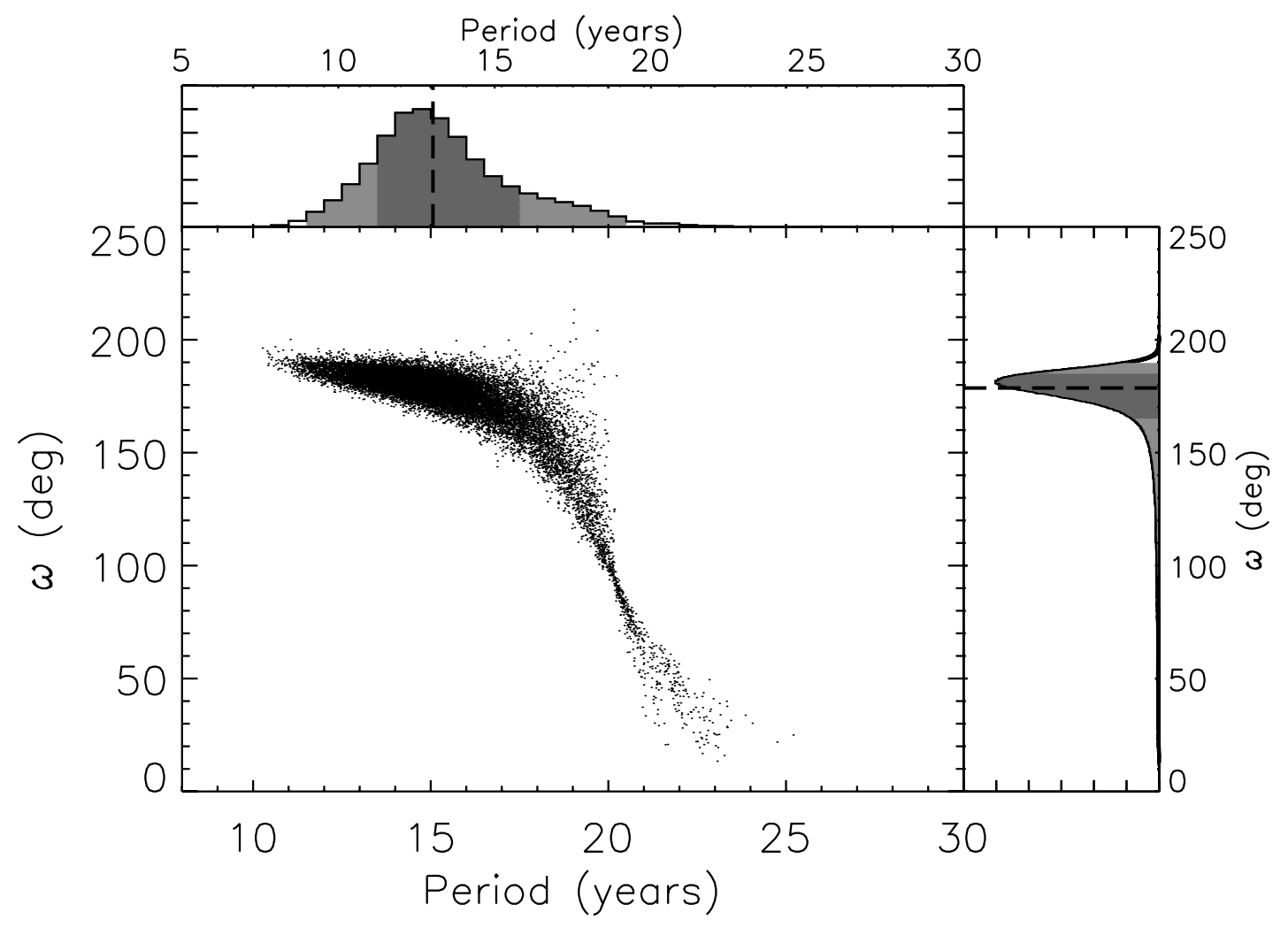

FIG. 7.- Results from MCMC determination of orbital period and the argument of periastron illustrating the degeneracy between these two parameters. The locus illustrates the two general branches of possible orbits, with short-period orbits having just completed apoastron and long-period orbits having just completed periastron. See Fig. 6 for further explanation.

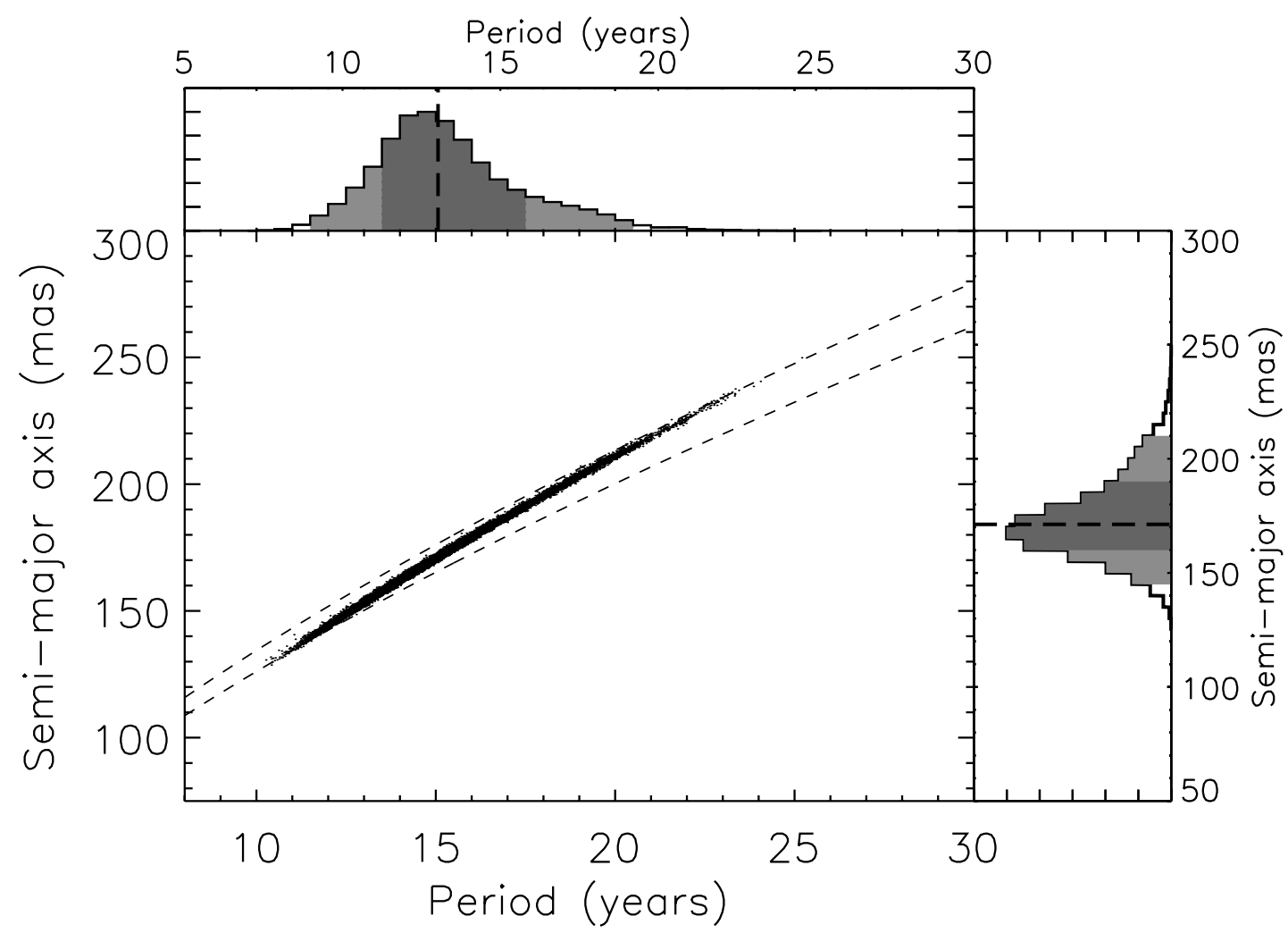

FIG. 8. - Results from MCMC determination of orbital period and semimajor axis. See Fig. 6 for further explanation. The dashed lines represent the $\pm 3 \sigma$ confidence intervals of the mass probability distribution. 


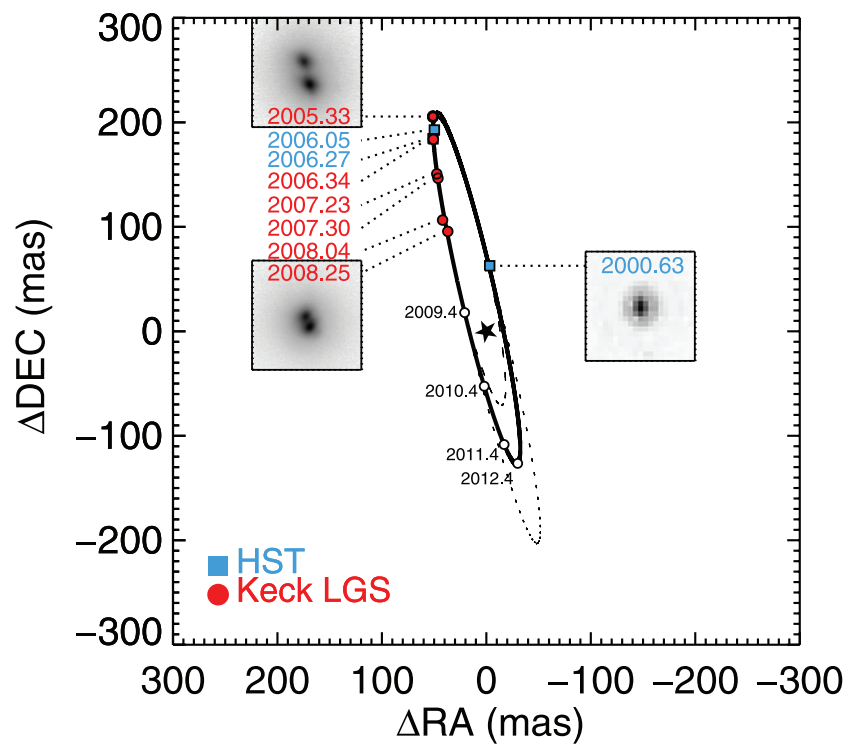

FIG. 9.-Keck LGS (red circles) and HST (blue squares) measurements of 2MASS J1534-2952AB along with three representative orbits from the MCMC fitting. Measurement errors are comparable to or smaller than the colored plotting symbols. The solid line shows the best-fitting orbit with a $15.2 \mathrm{yr}$ period, and the two dashed orbits represent alternative long-period (20 yr) and short-period (12 yr) solutions, chosen to represent the plausible range in period. The three orbits have reduced $\chi^{2}$ values of $0.9,1.0$, and 1.1. The open circles are the location of the secondary in future years as predicted by the $15.2 \mathrm{yr}$ period orbit. The image insets are $1^{\prime \prime}$ on a side, displayed with a square root stretch. (The HST WFPC2 image cutout has been rotated so that north is up. See Fig. 1 for the most accurate representation of the original data.) orbit means that it can be difficult to clearly distinguish whether the system has just passed apoastron or periastron, and thus a minority of the MCMC steps ( $2 \%$ ) fall into the long-period branch. ${ }^{22}$ Figure 8 shows the tight correlation between the semimajor axis and period.

The MCMC fitting provides probability distributions for the orbital parameters but does not provide a single best-fitting orbit per se, since a range of possible orbits fit the data with similar $\tilde{\chi}^{2}$ values. One illustration of this is the result for $\omega=179_{-14(83)}^{+6(11)} \mathrm{deg}$, where the $95 \%$ confidence limits are broad enough to span both the short-period and long-period solution branches. Thus, to plot orbits on the sky, we employ gradient-descent methods to find the best-fitting orbit with the MCMC-derived values as the starting point. Figures 9 and 10 show the resulting orbit, which has a period of $15.2 \mathrm{yr}$, a total mass of $0.0556 M_{\odot}$, and $\tilde{\chi}^{2}=0.9$. To illustrate how the uncertainty in the orbital period impacts the orbit, we also show the best-fitting orbits found when fixing the period to 12 and $20 \mathrm{yr}$, which have total masses of 0.0523 and $0.0590 M_{\odot}$ and $\tilde{\chi}^{2}$ of 1.1 and 1.0, respectively. All three orbits reside in the short-period branch and show that the projected separation is now rapidly decreasing. The system is expected to be well resolved again in the year 2011.

Applying Kepler's third law to the period and semimajor axis distributions gives the posterior probability distribution for the total mass of the binary, with median of $0.0556 M_{\odot}$, a standard deviation of $0.0018 M_{\odot}(3.2 \%)$, and a $68 \%(95 \%)$ confidence range of about $\pm 0.0018(0.0037) M_{\odot}$ (Fig. 11). However, the MCMC probability distribution does not include the uncertainties in the parallax $(1.6 \%)$ and the NIRC2 pixel scale $(0.11 \%)$. By Kepler's third law,

\footnotetext{
${ }^{22}$ Our inferred orbital period distribution is significantly longer than the $4 \mathrm{yr}$ estimate of Burgasser et al. (2003b). Their original estimate was based on the projected physical separation at the discovery epoch, an assumed total mass of $0.07 M_{\odot}$, and the statistical estimate from Fischer \& Marcy (1992) that the true semimajor axis is on average 1.26 times larger than the projected separation. The large discrepancy with our orbital period determination is not surprising, since a single epoch of imaging provides a highly uncertain period estimate: the likely true periods can span a factor of several greater or smaller (e.g., Torres 1999).
}
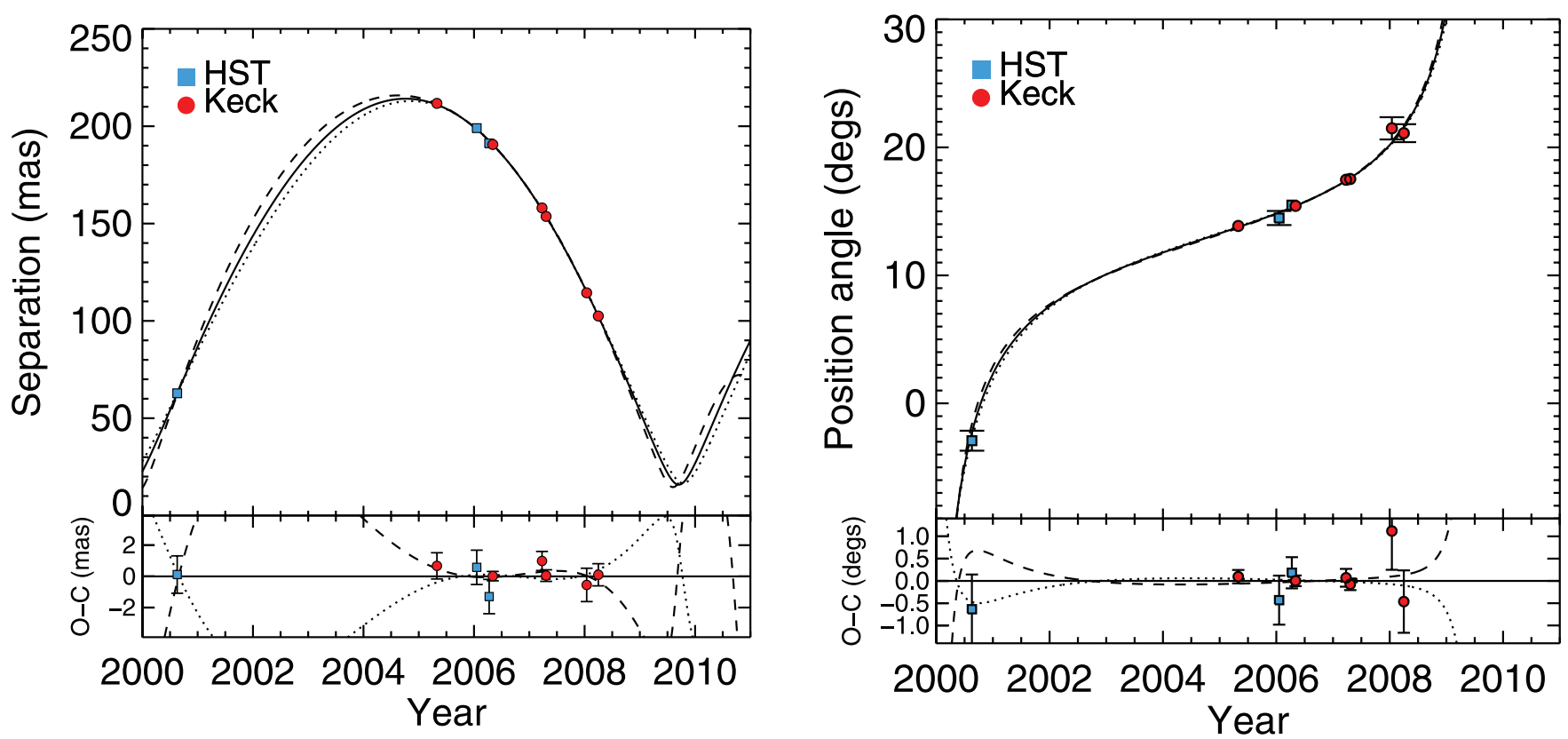

Fig. 10.-Keck LGS and HST measurements of 2MASS J1534-2952AB's separation (left) and P.A. (right), along with the three orbits plotted in Fig. 9. The measurement errors are comparable to or smaller than the plotting symbols, except for the three data points shown in the P.A. plot. The bottom panel of each plot shows the difference of the observed astrometry and the best-fitting (15.2 yr period) orbit. 


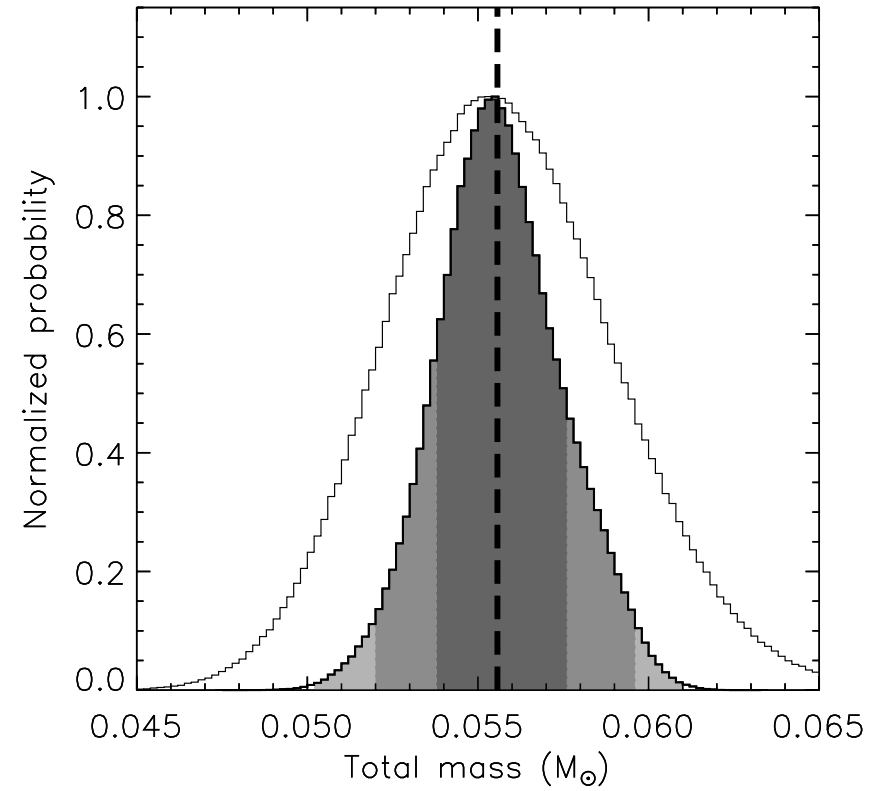

FIG. 11.- Total mass distribution from our MCMC analysis. The histogram is shaded to indicate the derived $68.3 \%, 95.5 \%$, and $99.7 \%$ confidence regions, which correspond to 1,2 , and $3 \sigma$ for a normal distribution. The solid vertical line represents the median value of $0.0556 M$. The standard deviation of the distribution is $0.0018 M_{\odot}$. The wider, unshaded histogram shows the final mass distribution, after accounting for the additional $4.9 \%$ error due primarily to the uncertainty in the binary's parallax: the result is essentially Gaussian with a standard deviation of $0.003 M_{\odot}$. The confidence limits for both distributions are given in Table 4 .

the quadrature sum of these errors amounts to an additional $4.9 \%$ uncertainty on the derived total mass. Since the MCMC-derived mass distribution is asymmetric, we account for this additional error in a Monte Carlo fashion; for each step in the chain, we draw a value for the pixel scale and parallax from a normal distribution and then compute the total mass. The resulting mass distribution is essentially Gaussian (Fig. 11). Our final determination of the total mass is $0.056 \pm 0.003(0.006) M_{\odot}$ at $68 \%(95 \%)$ confidence. Thus, the total mass of this system is well measured, with the parallax error being the dominant uncertainty. This is the coolest and lowest mass binary with a dynamical mass determination to date.

\subsubsection{Alternative Orbit Fits}

The WPFC2 discovery epoch in 2000 is obviously a key component to fitting the orbit. As described in $\S 2.2 .1$, we independently analyzed this data set and greatly reduced the measurement errors compared to those reported by Burgasser et al. (2003b). To examine the impact of this improvement, we also tried fitting the orbit using the original Burgasser et al. (2003b) astrometry. Without the 2008 Keck data, our improved WFPC2 astrometry is essential for a well-constrained fit. However, with the complete data set, the fitted orbital parameters and the total mass are insensitive to the specific choice of WFPC2 astrometry, changing by less than $1 \sigma$.

Also, to check the effect of our assumed prior on the MCMC fitting (flat in $\log P$ and $\log a$ ), we tried three alternative priors from the literature:

1. Solar-type stars.-Duquennoy \& Mayor (1991) analyzed a well-defined sample of 164 nearby solar-type stars (spectral type F7-G9) and found a lognormal distribution in orbital period:

$$
\frac{d N}{d(\log P)} \sim \exp \left[\frac{-(\log P-4.8)^{2}}{2 \sigma_{\log P}^{2}}\right]
$$

where $P$ is the period in days and $\sigma_{\log P}=2.3$.
2. Ultracool visual binaries. - Allen (2007) conducted a detailed analysis of published imaging surveys of 361 ultracool field objects to model the separation distribution as a lognormal distribution:

$$
\frac{d N}{d(\log a)} \sim \exp \left[\frac{-(\log a-0.86)^{2}}{2 \sigma_{\log a}^{2}}\right],
$$

where $a$ is the semimajor axis in AU and $\sigma_{\log a}=0.28 .^{23}$

3. Ultracool visual and spectroscopic binaries.-Maxted \& Jeffries (2005) analyzed a sample of 47 ultracool binaries with multiple radial velocity measurements and adopted a lognormal distribution truncated at large separations to match the known visual ultracool binaries:

$$
\frac{d N}{d(\log a)} \sim \exp \left[\frac{-(\log a-0.6)^{2}}{2 \sigma_{\log a}^{2}}\right] \text { for } a<10 \mathrm{AU}
$$

where $a$ is the semimajor axis in $\mathrm{AU}, \sigma_{\log a}=1.0$, and $d N / d(\log a)=0$ for $a>10 \mathrm{AU}$.

Figure 12 shows the posterior probability distributions for the total mass from the different priors. Overall, the choice of prior has very little effect on the mass determination. The Allen (2007) distribution favors slightly higher masses, but its results are consistent with the other priors.

As a final independent check, we also fit our astrometry using the linearized least-squares fitting routine ORBIT (Forveille et al. 1999), using the MCMC-derived parameters as the starting guess. The ORBIT results are given in Table 4 , with a resulting $\tilde{\chi}^{2}=0.9$, and show excellent agreement with the MCMC results.

\section{DISCUSSION}

A primary goal of measuring fundamental properties for ultracool binaries is to compare the measurements against theoretical models of their physical properties. A number of studies have been published for the previous ultracool visual binaries with dynamical masses $(\S 1)$, with subtle and/or overt differences in the ways that observations are compared to models. In the analysis that follows, we strive to clearly elucidate the comparison of our 2MASS J1534-2952AB observations to the models, in terms of both its approach and limitations. From the standpoint of the observations, we have high-quality measurements of (1) the total mass of the system and (2) the individual absolute magnitudes, with (3) the individual bolometric luminosities only slightly less reliable. (We have not measured the complete SED but have accounted for this uncertainty in computing $L_{\text {bol }}$ in $\S 3.2$.) We now

23 Since this distribution is derived from analysis of ultracool field dwarfs, one might arguably choose this as the default prior. However, Allen's input data are restricted to imaging and do not include any spectroscopic binaries; therefore, the smallest semimajor axes are poorly constrained. For instance, the Allen distribution predicts basically no binaries at $1 \mathrm{AU}$ or smaller separations ( $\gtrsim 3 \sigma$ events), perhaps at variance with spectroscopic binary studies (Maxted \& Jeffries 2005; Basri \& Reiners 2006). The young eclipsing M6.5+M6.5 binary 2MASS J0535-05 (Stassun et al. 2006) is also highly anomalous with this distribution ( $\sim 8 \sigma$ event). Finally, the Allen analysis does not separately consider objects of different spectral types, whereas Burgasser et al. (2006c) have suggested that the separation distribution for $\mathrm{T}$ dwarfs may be tighter than for the $\mathrm{L}$ dwarfs. Thus, our default prior of flat in $\log a$ is a conservative choice. Note that the longer period of 2MASS J1534-2952AB we find relative to the original estimate of $\sim 4 \mathrm{yr}(a \sim 1.1 \mathrm{AU})$ is in accord with the distribution proposed by Allen, which would indicate that such a $\sim 1.1$ AU system would be uncommonly rare $(\approx 3 \sigma)$ relative to the $\sim 100$ known ultracool binaries. 


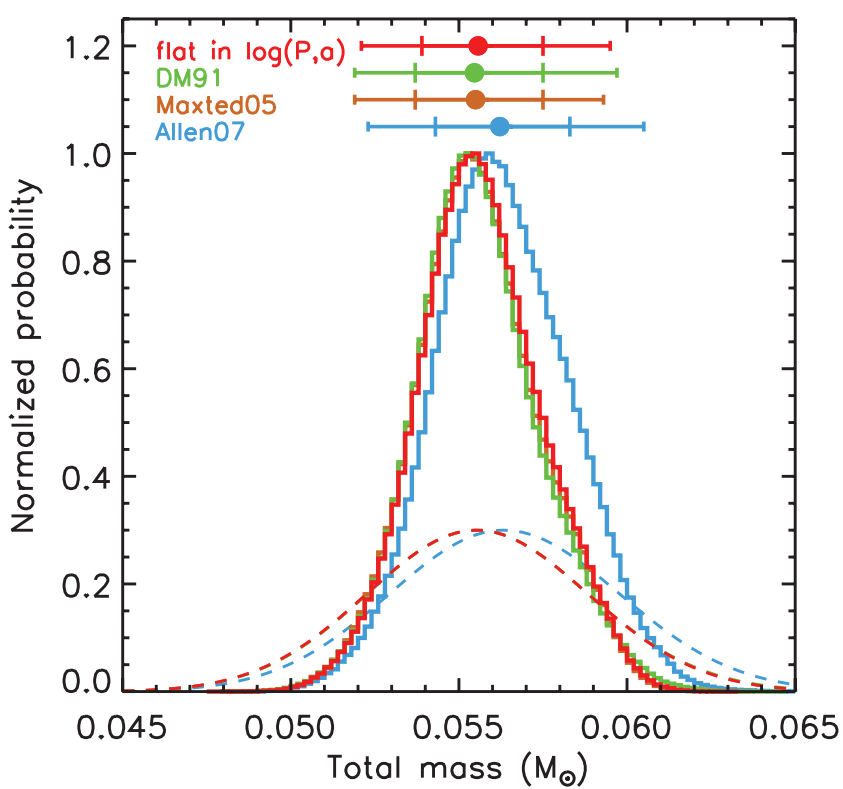

FIG. 12.- Total mass distribution from the MCMC analysis using four different priors: a distribution that is flat in the logarithm of the period and semimajor axis (our default assumption), the lognormal period distribution for solar-type stars from Duquennoy \& Mayor (1991), the lognormal semimajor distribution for field ultracool binaries from Allen (2007), and the truncated lognormal semimajor axis distribution from Maxted \& Jeffries (2005). The median value is indicated by the filled circle, and the two sets of errors bars indicate the $68.3 \%$ and $95.5 \%$ confidence regions, which correspond to 1 and $2 \sigma$ for a normal distribution. The dashed lines at the bottom show the resulting mass distributions after accounting for the uncertainty in the parallax. While the Allen (2007) prior favors slightly higher masses, all four priors give very consistent results.

examine what can be learned from these data in concert with evolutionary models and theoretical atmospheres.

\subsection{Substellarity}

The most immediate result from our measurement is that 2MASS J1534-2952AB is a bona fide brown dwarf binary. The total mass of $0.056 \pm 0.003 M_{\odot}$ is below the solar-metallicity stellar/substellar boundary of $\approx 0.070-0.074 M_{\odot}$ (e.g., Hayashi \& Nakano 1963; Kumar 1963; Burrows et al. 2001), with the boundary increasing to higher masses for lower metallicities (Saumon et al. 1994); therefore, the individual components are clearly substellar. This is the second binary where both components are directly confirmed to be brown dwarfs, after the young eclipsing M6.5+M6.5 binary 2MASS J0535-05 (Stassun et al. 2006), and is the first such field binary in this category.

\subsection{Age}

Brown dwarfs follow a mass-luminosity-age relation. We have measured two of these quantities, the (total) mass and the luminosity, and by using evolutionary tracks we can determine the third quantity, the age of the system. We use models from the Tucson group (Burrows et al. 1997), which provide predictions for $L_{\text {bol }}$, and the "COND" models from the Lyon group (Baraffe et al. 2003), which predict both $L_{\text {bol }}$ and absolute magnitudes. We conservatively assume that the system is coeval and is a true binary, not a partially (un)resolved higher order multiple system $(\S 3.1)$.

For each tabulated model age, we use the individual absolute magnitudes and/or bolometric luminosities to calculate the mass of the components and then sum the masses. We then apply the observed total mass to determine the age range of the system. All measurement errors in $L_{\mathrm{bol}}$ and the total mass are accounted for in

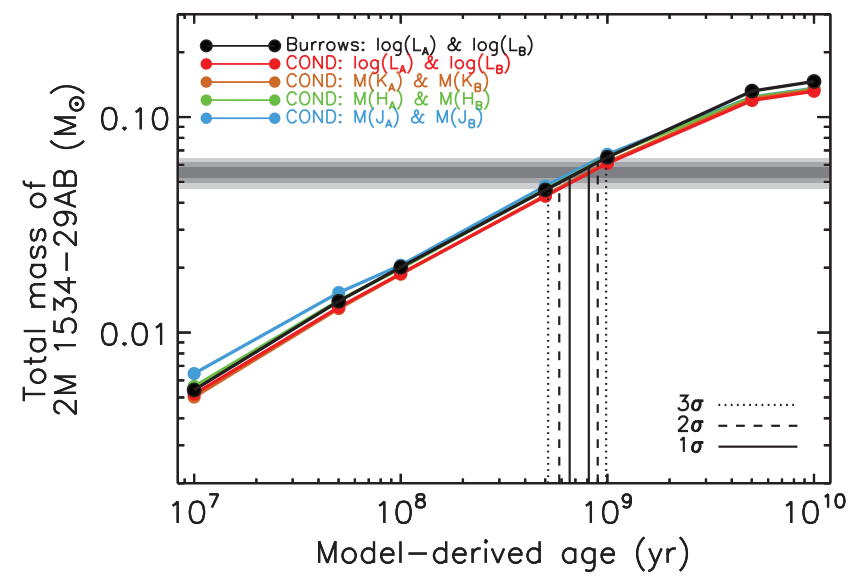

FIG. 13.-Determination of the age of the 2MASS J1534-2952AB system from evolutionary models using the observed magnitudes / luminosity and total mass. Two sets of models are employed: the models from Burrows et al. (1997) and the COND models from Baraffe et al. (2003). The horizontal gray bars indicate our measured 1,2, and $3 \sigma$ constraints on the total mass, and the vertical lines show the corresponding ages derived from the Burrows et al. (1997) models. Both sets of models produce consistent results, given in Table 5. The COND models provide predictions for both the absolute magnitudes and bolometric luminosity, so all of these are shown. Note that these plotted lines are computed for an object with the fluxes of 2MASS J1534-2952AB and are not generally applicable to other binaries. See $\S 4.2$ for details.

a Monte Carlo fashion, namely, we repeat the model calculations over multiple realizations for the $L_{\mathrm{bol}}$ and the total mass values. We take great care to account for the covariance between the relevant quantities in the calculation. For instance, the total mass of the system and the luminosity both depend on the parallax, and thus their errors are positively correlated; we therefore draw the parallax values from a normal distribution and incorporate these in determining the Monte Carlo distribution of total masses and luminosities, which themselves are then propagated in the model-based calculations. This approach results in a probability distribution for the system's age (as well as the other resulting parameters discussed below), which we summarize with the median value and confidence limits.

Figure 13 shows the results of these calculations to determine the age of the system. For a consistent comparison between the Lyon and Tucson models, we use only the results derived from the $L_{\text {bol }}$ measurements. However, the figure also shows that using the absolute magnitudes predicted by the Lyon models would give similar results.

We determine an age of $0.73 \pm 0.07(0.15)$ Gyr from the Burrows models and $0.83 \pm 0.08(0.18)$ Gyr from the Baraffe models at $68 \%(95 \%)$ confidence. To construct a representative "average" of the model results, we merge the results of the individual Monte Carlo calculations into a single distribution and compute its confidence limits. Thus, we assign an age of $0.78 \pm$ $0.09(0.18)$ Gyr ( Table 5). This is relatively youthful compared to the main-sequence stars in the solar neighborhood, e.g., $\approx 95 \%$ of nearby solar-type stars have age estimates of $\gtrsim 1$ Gyr (Nordström et al. 2004). However, the mean age of T dwarfs is expected to be younger than for field stars, since the known census is magnitude limited and younger objects are brighter. The age distribution of field ultracool dwarfs has been modeled by Burgasser (2004) and Allen et al. (2005); they generally find that field T dwarfs can span younger ages than for low-mass stars, although the predicted age distributions for both types of objects have large spreads.

Kinematics provides an independent (albeit indirect) indicator of age, as older objects are expected to generally show larger 
TABLE 5

Evolutionary Model-derived Properties of 2MASS J1534-2952AB

\begin{tabular}{|c|c|c|c|c|c|c|}
\hline \multirow[b]{2}{*}{ Property } & \multicolumn{2}{|c|}{ Tucson Models } & \multicolumn{2}{|c|}{ Lyon Models } & \multicolumn{2}{|c|}{ "Average" } \\
\hline & Component $\mathrm{A}$ & Component B & Component $\mathrm{A}$ & Component B & Component A & Component B \\
\hline $\log ($ age $) \ldots \ldots \ldots \ldots$ & \multicolumn{2}{|c|}{$8.86 \pm 0.04(0.09)$} & \multicolumn{2}{|c|}{$8.92 \pm 0.04(0.09)$} & \multicolumn{2}{|c|}{$8.89 \pm 0.05(0.10)$} \\
\hline 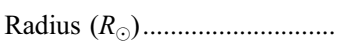 & $0.0997_{-0.0012(0.0024)}^{+0.0012(0.0025)}$ & $0.1004 \pm 0.0012(0.0024)$ & $0.0978_{-0.0010(0.0022)}^{+0.0010(0.021)}$ & $0.0984_{-0.0012(0.0025)}^{+0.0011(0.022)}$ & $0.0986 \pm 0.0015(0.0028)$ & $0.0993 \pm 0.0017(0.0031)$ \\
\hline 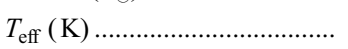 & $1019_{-15(31)}^{+0.60(32)}$ & $970_{-15(29)}^{+15(31)}$ & $1034_{-15(30)}^{+16(32)}$ & $985_{-15(29)}^{+15(32)}$ & $1028 \pm 17(35)$ & $978 \pm 17(34)$ \\
\hline $\log g(\mathrm{cgs}) \ldots \ldots \ldots \ldots$ & $4.90 \pm 0.04(0.07)$ & $4.86 \pm 0.04(0.07)$ & $4.92 \pm 0.04(0.07)$ & $4.88_{-0.04(0.07)}^{+0.04)}$ & $4.91 \pm 0.04(0.07)$ & $4.87 \pm 0.04(0.07)$ \\
\hline$\Delta T_{\text {eff }}(\mathrm{K})$ & \multicolumn{2}{|c|}{$50_{-10(19)}^{+6(10)}$} & \multicolumn{2}{|c|}{$50_{-10(19)}^{+6(10)}-^{-0.04(0.01)}$} & \multicolumn{2}{|c|}{$50^{+6(10)}$} \\
\hline Mass ratio $M_{\mathrm{B}} / M_{\mathrm{A}} \ldots \ldots \ldots \ldots \ldots$ & \multicolumn{2}{|c|}{$0.937_{-0.007(0.012)}^{+0.012(0.024)}$} & \multicolumn{2}{|c|}{$0.934_{-0.008(0.013)}^{+0.012(0.025)}$} & \multicolumn{2}{|c|}{$0.936_{-0.008(0.013)}^{+0.012(0.024)}$} \\
\hline 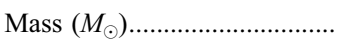 & $0.0287_{-0.0016(0.0032)}^{+0.0017(0.0036)}$ & $0.0270_{-0.0015(0.0030)}^{+0.0016(0.003)}$ & $0.0288_{-0.0016(0.0031)}^{+0.0017(0.0035)}$ & $0.0269_{-0.0016(0.0030)}^{+0.0017(0.0034)}$ & $0.0287 \pm 0.0016(0.0033)$ & $0.0269 \pm 0.0016(0.0032)$ \\
\hline
\end{tabular}

Notes.-Median values of physical parameters derived for the two components of 2MASS J1534-2952AB from the evolutionary models along with the $68 \%(95 \%)$ confidence limits, as described in $\S 4.2$. By construction, the ages of the two components are identical for a given set of models, since the analysis assumes that the system is coeval, and thus a single model-derived age is listed for both components. Note that the two sets of models predict nearly identical mass ratios so the two sets of individual masses are the same. 
space motions due to their accumulated history of dynamical interactions (e.g., Wielen 1977). The tangential velocity of 2MASS $\mathrm{J} 1534-2952 \mathrm{AB}\left(V_{\tan }=17.3 \pm 0.4 \mathrm{~km} \mathrm{~s}^{-1}\right)$ is the second smallest measured for T dwarfs, with only the T5.5 dwarf 2MASS 15463325 being smaller $\left(12.1 \pm 0.4 \mathrm{~km} \mathrm{~s}^{-1}\right.$; Tinney et al. 2003). This is generally in accord with the $0.78 \pm 0.09$ Gyr inferred from the evolutionary models, namely, that 2MASS J1534-2952AB is among the youngest members of the nearby field population. However, since the measured $V_{\tan }$ distribution of field T dwarfs is quite broad, with an unweighted average of $38.4 \mathrm{~km} \mathrm{~s}^{-1}$ and a standard deviation of $20.4 \mathrm{~km} \mathrm{~s}^{-1}$ among the 21 unique objects in the Tinney et al. (2003) and Vrba et al. (2004) parallax samples, 2MASS J1534-2952AB does not appear to be anomalously young for a field object. A radial velocity measurement is needed to determine the binary's space motion and thus better constrain its kinematics.

\subsection{Temperatures and Surface Gravities}

With the age of the system determined above, the combination of the observations and the evolutionary models provides highly precise values for the remaining physical parameters. The results derived from the two sets of evolutionary tracks are given in Table 5 and are computed from the same Monte Carlo approach that accounts for the covariance in the measurements. The Tucson and Lyon models give consistent values, with the Tucson models giving slightly larger radii and thus slightly cooler temperatures. Again, to compute a representative "average" for each parameter, we merge the Monte Carlo distributions computed from each set of models and compute confidence limits for the aggregate. This is not intended to be physically meaningful, but rather is a quantitative representation of the results that accounts for non-Gaussian and/or inconsistent distributions from the two sets of models. We thus find radii of $0.0986 \pm 0.0015$ and $0.0993 \pm 0.0017 R_{\odot}$, effective temperatures of $1028 \pm 17$ and $978 \pm 17 \mathrm{~K}$, and surface gravities of $\log g=4.91 \pm 0.04$ and $4.87 \pm 0.04$ for components $\mathrm{A}$ and $\mathrm{B}$, respectively. ${ }^{24}$

To reiterate, these properties are derived using only the measured total mass and resolved magnitudes/luminosities, along with the assumption that the system is coeval and composed of two components. No additional assumptions have been made to determine the individual masses. We also have avoided using spectral types and/or effective temperatures in this aspect of our analysis (contrary to some previous studies), as these quantities can introduce additional systematic errors and/or circular reasoning. For instance, it would be incorrect to employ the relations between spectral type and $T_{\text {eff }}$ from Golimowski et al. (2004a) or Vrba et al. (2004) to determine $T_{\text {eff }}$ for the two components and then compare to evolutionary tracks, as the Vrba et al. (2004) and Golimowski et al. (2004a) relations are derived from the radii of field brown dwarfs predicted by the evolutionary tracks themselves. Likewise, it is not necessary to use $T_{\text {eff }}$ determinations from atmospheric models to determine the age of the system or the physical properties of the individual components in our approach (see also $\S 4.5)$.

As already noted above, our Monte Carlo calculations account for the covariance in the measurements. One important effect is that the uncertainties derived from the resolved magnitudes and luminosities of the two components are correlated, since they all depend on the measurement uncertainties in the integrated-light photometry of the system. As a consequence, the relative temper-

\footnotetext{
24 Since all these derived properties rely on the model-derived age, their distributions from the Monte Carlo calculations are fairly correlated. However, the formal confidence limits on the quantities are so small that this correlation is unlikely to be significant for any future analyses.
}

ature difference between the two components $\left(\Delta T_{\text {eff }}=50_{-10}^{+6} \mathrm{~K}\right)$ can be calculated to higher precision than would be indicated by the uncertainties in the individual $T_{\text {eff }}$ determinations $(\sqrt{2} \times$ $17 \mathrm{~K}=24 \mathrm{~K})$. This $\Delta T_{\text {eff }}$ agrees with the $\approx 70 \mathrm{~K}$ difference expected from the Golimowski et al. (2004a) polynomial fits for the 0.5 subclass difference between the two components. (The $\Delta T_{\text {eff }}$ from the Golimowski et al. [2004a] fits would be about twice as large for a 1 subclass difference.)

The physical parameters for 2MASS J1534-2952A and 2MASS J1534-2952B are in general agreement with previous determinations for the properties of field $\mathrm{T}$ dwarfs. However, our values have much higher precision because the accurate total mass measurement leads to a small age range, which leads to strong constraints on the radii and thus small uncertainties on the derived $T_{\text {eff }}$ and $\log g$ values. (We discuss this further in $\S$ 4.5.) $\mathrm{T}$ dwarf surface gravities have been inferred to be $\log g=4.5-5.5$ by comparing theoretical model atmospheres to optical spectra (Burrows et al. 2002), near-IR colors and line strengths (Knapp et al. 2004), and low-resolution near-IR / mid-IR spectra (Burgasser et al. 2006a; Saumon et al. 2007; Leggett et al. 2007a; Cushing et al. 2008). This range encompasses our $\log g$ determinations for 2MASS J1534-2952AB.

On the other hand, our very precise temperatures for the two components of 2MASS J1534-2952AB are discrepant with previous studies of T dwarfs: the $T_{\text {eff }}$ values of 2 MASS J15342952AB appear to be cooler than determined previously for mid-T dwarfs. These discrepancies occur for two separate comparisons.

\subsubsection{Temperature Discrepancy with Evolutionary Models}

Temperatures for field T dwarfs have been inferred by combining accurate $L_{\text {bol }}$ determinations with radius predictions from evolutionary tracks. This approach is expected to be reasonably accurate, since the radii of brown dwarfs older than $\sim 100 \mathrm{Myr}$ are predicted to vary by $\$ 30 \%$. Golimowski et al. (2004a) adopt a typical age of 3 Gyr and a plausible range of $0.1-10 \mathrm{Gyr}$ in computing $T_{\text {eff }}$ from $L_{\text {bol }}$, and Vrba et al. (2004) adopt a radius range of $0.90 \pm 0.15 R_{\mathrm{J}}$ predicted by Burgasser (2002) simulations of the solar neighborhood assuming a constant star formation history. (Both studies use the Tucson evolutionary tracks.) There are eight T4.5-T6.5 dwarfs in these studies (after updating the spectral types to the latest classification by Burgasser et al. $2006 \mathrm{~b}$ ), and all of these appear to be single based on high angular resolution imaging (Burgasser et al. 2003b, 2006c; M. C. Liu et al. 2008 , in preparation). We compute the average and standard deviation of both studies to obtain $T_{\text {eff }}=1216 \pm 20 \mathrm{~K}$ for T4.5 (two objects), $1146 \mathrm{~K}$ for T5.0 (interpolated), $1077 \mathrm{~K}$ for T5.5 (one object), $1014 \pm 33 \mathrm{~K}$ for T6.0 (three objects), and $950 \pm$ $106 \mathrm{~K}$ for T6.5 (three objects). We have excluded the T6+T8 binary 2MASS J1225-2739AB and the peculiar T6 dwarf 2MASS $\mathrm{J} 0937+2931$, and we have assumed that the T4.5 dwarf 2MASS J0559-1404 is an equal-magnitude unresolved binary based on its pronounced overluminosity (e.g., Fig. 3 of Burgasser 2007b). Neither sample contains any T5.0 objects, but the four other subclasses almost exactly follow a straight line, so we linearly interpolate to find $T_{\text {eff }}$ for T5.0.

In comparison to the field objects, the components of $2 \mathrm{MASS}$ J1534-2952AB appear to have $\approx 100 \mathrm{~K}$ cooler temperatures relative to their spectral subclass $(\approx 120 \pm 35 \mathrm{~K}$ for the primary and $\approx 100 \pm 35 \mathrm{~K}$ for the secondary, where we have adopted a $30 \mathrm{~K}$ uncertainty for the T5 and T5.5 field objects based on the other subclasses with more than one object). The disagreement is modest, and a more definitive comparison is hampered by the few $T_{\text {eff }}$ determinations (i.e., parallaxes) for T4.5-T6.5 dwarfs. Nevertheless, the result is potentially intriguing. 
In particular, Metchev \& Hillenbrand (2006) have noted perhaps a similar effect for the three known late L (L7-L8) dwarf companions to field stars. More precise $T_{\text {eff }}$ estimates can be obtained from evolutionary models for these companions than for field objects by incorporating the age estimates of their primary stars (see $\S 4.5$ for details). Metchev \& Hillenbrand (2006) find that the L dwarf companions appear to be $\approx 100-200 \mathrm{~K}$ cooler than single-field late L dwarfs. They raise the possibility that the modelderived radii are at fault, due to either incorrect cooling rates or systematic overestimate of the field dwarf ages. However, they prefer the hypothesis that the discrepancy is a manifestation of an unanticipated surface gravity dependence of the L/T transition, causing younger L/T transition objects to have cooler temperatures than older ones. This is motivated by their analysis of the young (0.1-0.4 Gyr) L7.5 companion HD 203030B and apparently supported by the young $(0.1-0.5$ Gyr) T2.5 companion HN Peg B, which also appears to be $\approx 200 \mathrm{~K}$ cooler than field objects of the same spectral type (Luhman et al. 2007; Leggett et al. 2008).

We find that the T5.0 and T5.5 components of 2MASS J1534$2952 \mathrm{AB}$ may also be $\approx 100 \mathrm{~K}$ cooler than comparable field objects. Since these two components are later type than the L/T transition (e.g., their positions in IR color-magnitude diagrams are coincident with the locus of $\mathrm{mid} /$ late $\mathrm{T}$ dwarfs with blue near-IR colors), this may suggest that the $T_{\text {eff }}$ discrepancy might not be solely associated with the L/T transition. Instead, 2MASS J1534-2952AB and the aforementioned L/T companions may indicate that a systematic error in the estimated ages and radii of field late $\mathrm{L}$ and $\mathrm{T}$ dwarfs is the culprit.

The $\approx 10 \%$ temperature discrepancy for 2MASS J1534$2952 \mathrm{AB}$ amounts to $\mathrm{a} \approx 20 \%$ underestimate of the radii. For a fixed value of $L_{\text {bol }}$ (which is the appropriate constraint here), the Burrows et al. (2001) scaling relations give

$$
t \sim R^{-8.56}
$$

where $t$ is the age and $R$ is the radius. This agrees well with the exponent value of 8.2-8.4 extracted from the Tucson models for sources of $\log \left(L_{\mathrm{bol}} / L_{\odot}\right)=-5.0$ to -5.1 . Propagating the $\pm 3 \%$ uncertainties in the $T_{\text {eff }}$ disagreement, the implied age overestimate is a factor of $6 \pm 3$, meaning implied ages of $\approx 0.3-1.0 \mathrm{Gyr}$ for the field population. ${ }^{25}$

The same discrepancy can be seen in an alternate fashion, namely, by comparing the luminosities for the same T4.5-T6.5 field objects: $\log \left(L_{\mathrm{bol}} / L_{\odot}\right)=-4.79 \pm 0.04$ for T4.5, -4.89 for T5.0 (interpolated), $-4.99 \pm 0.01$ for T5.5, $-5.17 \pm 0.29$ for T6.0, and $-5.22 \pm 0.20$ for T6.5. The luminosities of 2MASS J1534-2952AB are comparable to the field objects of similar type $(-5.015 \pm 0.019$ for T5.0 component $A$ and $-5.093 \pm 0.019$ for T5.5 component B). Thus, in order for all the objects to have similar temperatures and $L_{\mathrm{bol}}$, they must have about the same radius and thus about the same age as 2MASS J1534-2952AB. In other words, the measured total mass of 2MASS J1534$2952 \mathrm{AB}$ is too small (by a factor of $\approx 2$ ) compared to the mass expected from the evolutionary models for 3 Gyr objects with $\log \left(L_{\mathrm{bol}} / L_{\odot}\right) \approx-5.0$.

A representative age of $\sim 0.5$ Gyr for the field population is not ruled out given the state of the observations. Although Golimowksi et al. (2004a) did consider the range of $0.1-10 \mathrm{Gyr}$, they adopted a nominal age of 3 Gyr in determining $T_{\text {eff }}$ for field dwarfs, based on the 2-4 Gyr age estimate from the tangential velocities of ultracool dwarfs by Dahn et al. (2002). A younger age could be ac-

\footnotetext{
25 Applying the same scaling relation to the $10 \%-15 \%$ radius discrepancy found by Metchev \& Hillenbrand (2006) for objects at the L/T transition implies a factor of $2-3$ overestimate in the representative age of the field population.
}

commodated, since the tangential velocity of a population is only an approximate statistical estimate of its age. Indeed, kinematic analysis of the space motions of $\mathrm{L}$ and $\mathrm{T}$ dwarfs suggests a younger age of $\approx 0.5-2$ Gyr (Zapatero Osorio et al. 2007). Similarly, the radii of $0.90 \pm 0.15 R_{\mathrm{J}}$ adopted by Vrba et al. (2004) are based on a mass function where $d N / d M \sim M^{-1}$; a somewhat steeper mass function would lead to younger typical ages (e.g., Fig. 8 of Burgasser 2004; although Metchev et al. [2008] suggest $d N / d M \sim$ $M^{0}$ based on a small sample of T dwarfs). Thus, the discrepancy of evolutionary model-derived temperatures between objects of known mass/age and the field population can be plausibly explained by a modest overestimate of the ages of the field population. A larger sample of ultracool dwarfs with known masses and/or ages is needed to better explore this issue $(\S 4.5)$.

\subsubsection{Temperature Discrepancy with Model Atmospheres}

The spectrum of 2MASS J1534-2952AB has not yet been fitted with model atmospheres due to its composite nature. In fact, the T dwarf class as a whole has not been extensively subjected to such comparisons. Burgasser et al. (2006a) determined $T_{\text {eff }}$ for a sample of 16 T5.5-T8 dwarfs by comparing near-IR spectral indices to condensate (dust)-free atmosphere models from the Tucson group. They determined $T_{\text {eff }}=1020-1100 \mathrm{~K}$ for one T5.5 dwarf, and an unweighted linear fit of atmosphere-derived $T_{\text {eff }}$ versus spectral type for their sample (excluding the peculiar T6 dwarf 2MASS J0937+2931) gives

$$
T_{\text {eff }, \text { atmosphere }}=1090-126(\mathrm{SpT}-25.5),
$$

where $\mathrm{SpT}=25.5$ for $\mathrm{T} 5.5, \mathrm{SpT}=26$ for $\mathrm{T} 6$, etc. The rms about the linear fit is $50 \mathrm{~K}$, which we adopt as the uncertainty (a value somewhat larger than the \pm 10 to $\pm 40 \mathrm{~K}$ range computed for individual objects in their sample). Extrapolating the linear fit gives $T_{\text {eff }}=1160 \mathrm{~K}$ for T5. This is obviously approximate, e.g., given the potential systematic effects in the models and the spectral classification scheme, although this value agrees with the $T_{\text {eff }}=1150-1200 \mathrm{~K}$ found by fitting model atmospheres to the $0.95-14.5 \mu \mathrm{m}$ spectrum of the T4.5 dwarf 2MASS J0559-1404 (Cushing et al. 2008).

Thus, model atmospheres indicate $T_{\text {eff }}=1160 \pm 50$ and $1090 \pm$ $50 \mathrm{~K}$ for 2MASS J1534-2952A and 2MASS J1534-2952B, respectively. The temperatures we find using evolutionary tracks appear to be cooler by $\approx 100 \mathrm{~K}$ at modest significance $(-130 \pm$ $50 \mathrm{~K}$ for component $\mathrm{A}$ and $-110 \pm 50 \mathrm{~K}$ for component $\mathrm{B}$, if we assume that the errors add in quadrature). We cannot objectively discern if the problem lies in the evolutionary tracks, the model atmospheres, or both. However, the evolutionary models are thought to be robust to the principal input uncertainties (Chabrier et al. 2000). On the other hand, the model atmospheres are quite uncertain. Even though the spectral appearance of mid- and late T dwarfs is relatively simple, dominated by collision-induced $\mathrm{H}_{2}$, $\mathrm{H}_{2} \mathrm{O}$, and $\mathrm{CH}_{4}$ in the near-IR and the wings of the $\mathrm{K}$ I $0.77 \mu \mathrm{m}$ resonance line in the far-red, the line lists for $\mathrm{H}_{2} \mathrm{O}$ and $\mathrm{CH}_{4}$ are known to be incomplete, and the input physics to the atmosphere models are complex. Current atmospheres generally match the observed spectra of late T (T6-T8) dwarfs, but not exactly so (e.g., Burrows et al. 2006; Burgasser et al. 2006a; Saumon et al. 2007; Leggett et al. 2007a). ${ }^{26}$ Therefore, while a larger sample of objects is needed for both dynamical mass determinations and model atmosphere fitting, the plausible hypothesis is that the

\footnotetext{
26 For instance, recognizing these limitations, Burgasser et al. (2006a) chose to calibrate the model atmosphere predictions empirically using the well-studied T7.5 dwarf G1 570D in fitting models to late T dwarf spectra, as opposed to using the atmospheres directly.
} 

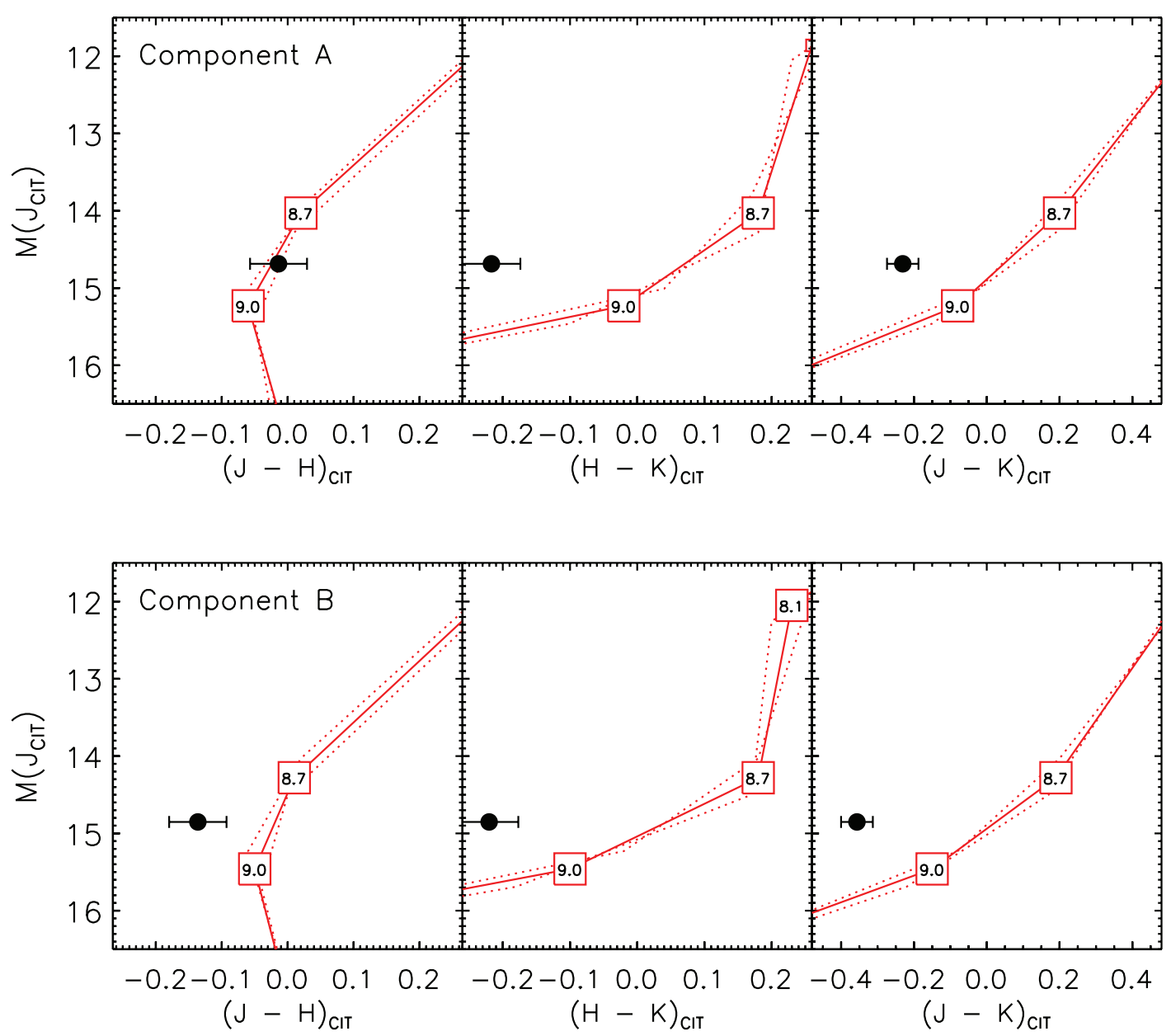

FIG. 14. - Color-magnitude diagrams for the individual components of 2MASS J1534-2952AB compared to the Lyon COND models (Baraffe et al. 2003), on the CIT photometric system. The models corresponding to the individual component masses and their $\pm 1 \sigma$ range are plotted as solid lines and dotted lines, respectively. The numbered boxes indicate the logarithm of the model age in Gyr. The error bars on the absolute $J$-band magnitude are smaller than the plotting symbol. The models do not match the data very well, which can be ascribed to the incomplete opacities in the model atmospheres.

observed discrepancy arises from an overprediction of $T_{\text {eff }}$ by current model atmospheres.

\subsection{Color-Magnitude and Hertzsprung-Russell Diagrams}

We have directly measured the total mass of the 2MASS J15342952AB system. However, using the evolutionary tracks to determine the physical properties also implicitly determines the mass ratio, since the model-derived age and observed individual luminosities translate into individual masses (again with the assumption that the system is composed of only two components). We infer the mass ratio of the system from the ratio of the bolometric luminosities, as this is very robust. To illustrate this, consider the analytic scaling relation for solar-metallicity substellar objects from Burrows et al. (2001):

$$
L_{\mathrm{bol}} \sim M^{2.64} t^{-1.3} \kappa_{\mathrm{R}}^{0.35},
$$

where $M$ is the mass, $t$ is the age, and $\kappa_{\mathrm{R}}$ is the Rosseland mean opacity. We measure a $0.078 \pm 0.016$ dex difference in $L_{\mathrm{bol}}$ between the two components, ${ }^{27}$ which leads to a mass ratio

\footnotetext{
27 At face value, the results in Table 3 would give an uncertainty of $\sqrt{2} \times 0.018=0.025$ dex in the $L_{\text {bol }}$ difference, but this would include the uncertainties in the distance modulus $(0.04 \mathrm{mag})$ and the integrated-light photometry (0.03 mag), which are common to both components.
}

$q \equiv M_{\mathrm{B}} / M_{\mathrm{A}}=0.934 \pm 0.007$. The uncertainty in the mass ratio is small due to the weak dependence of mass on luminosity at fixed age. Using the actual tabulated Tucson and Lyon models and again keeping careful track of the covariance in the calculations, we compute a final value of $q=0.936_{-0.008}^{+0.012}$, where the error includes the uncertainties in the model-inferred age and the observed $L_{\mathrm{bol}}$ difference. (The Tucson and Lyon models give basically identical results for $q$.) This gives individual masses of $0.0287 \pm 0.0016 M_{\odot}$ $\left(30.1 \pm 1.7 M_{\mathrm{J}}\right)$ and $0.0269 \pm 0.0016 M_{\odot}\left(28.2 \pm 1.7 M_{\mathrm{J}}\right)$ for components $\mathrm{A}$ and $\mathrm{B}$, respectively.

We first compare the individual components against the COND evolutionary models of the Lyon group, which provide predictions for the absolute magnitudes and colors. The model predictions are generated for the CIT photometric system, so we transform our resolved MKO photometry for 2MASS J1534-2952AB to this system using the results of Stephens \& Leggett (2004). Figure 14 shows that the models are somewhat too red compared to the data. This is not surprising, as model atmospheres for $\mathrm{T}$ dwarfs are known to be deficient in the $\mathrm{CH}_{4}$ and $\mathrm{H}_{2} \mathrm{O}$ opacities relevant at these wavelengths (e.g., Leggett et al. 2007a). The plotted COND models are also computed only for solar metallicity, and a nonsolar metallicity for 2MASS J1534-2952AB would impact the colors and magnitudes (e.g., Liu et al. 2007; Burgasser 2007a). Indeed, current models do not exactly match the observed colormagnitude loci for field T dwarfs (e.g., Fig. 8 of Knapp et al. 


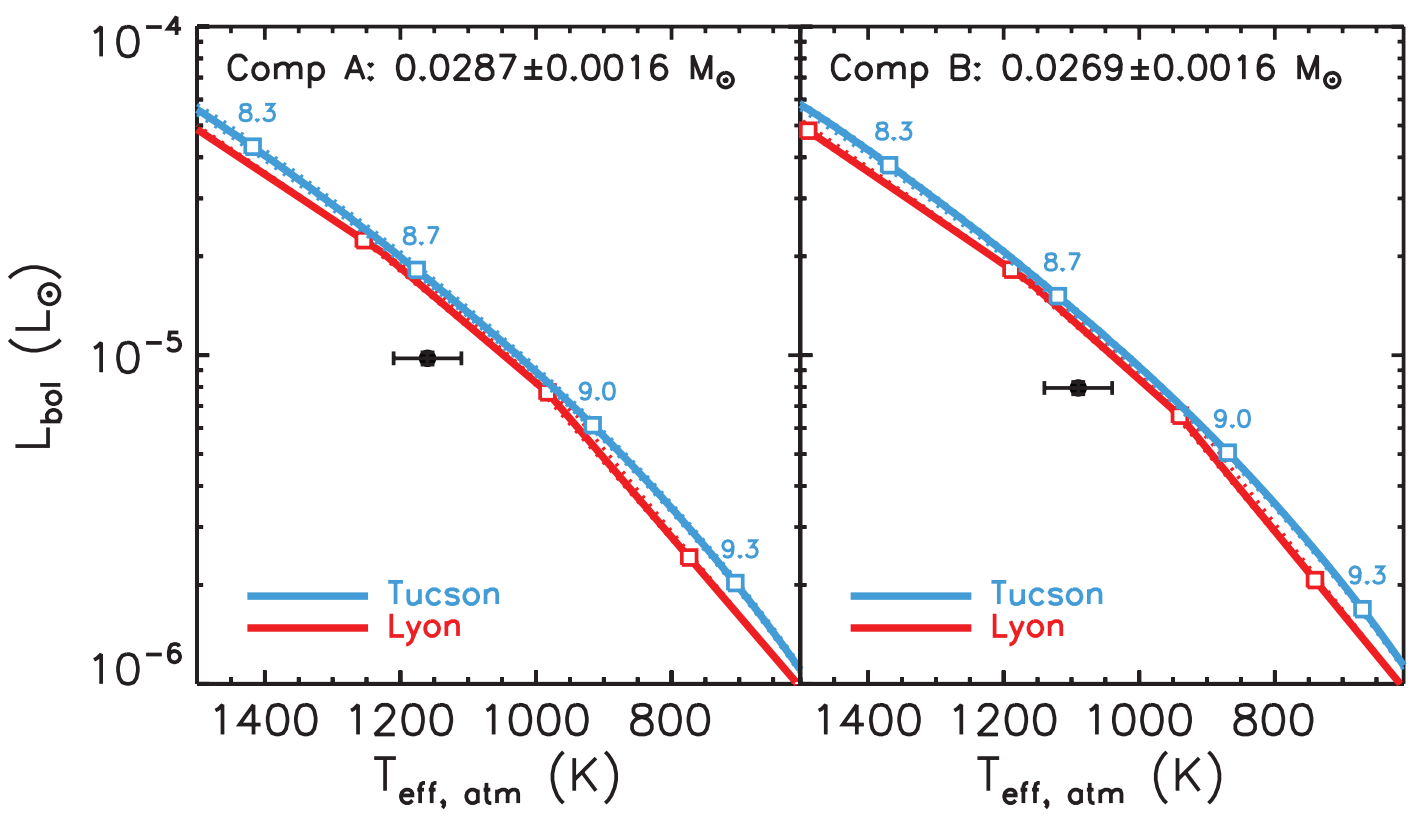

FIG. 15.-H-R diagram showing the individual components of 2MASS J1534-2952AB compared to evolutionary tracks from the Tucson and Lyon groups. The

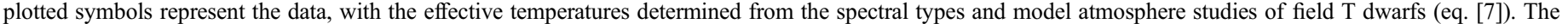

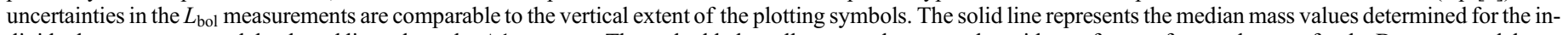

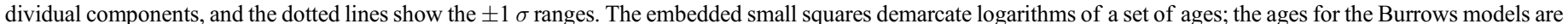

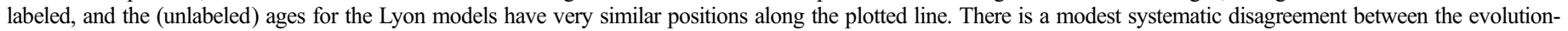
ary tracks and observations.

2004; Fig. 7 of Burrows et al. 2006). Nevertheless, 2MASS J1534$2952 \mathrm{AB}$ will provide a strong test for future models, since the components' magnitudes, colors, and masses are very well measured.

With the individual mass estimates and an independent determination of $T_{\text {eff }}$, it is possible to directly test different evolutionary tracks using the Hertzsprung-Russell (H-R) diagram. We use the values of $T_{\text {eff }}=1160 \pm 50$ and $1090 \pm 50 \mathrm{~K}$ for $2 \mathrm{MASS}$ J1534-2952A and 2MASS J1534-2952B, respectively, derived in $\S 4.3$ from model atmosphere studies. Figure 15 shows the individual components on the H-R diagram and compares these to the Tucson and Lyon evolutionary tracks. The locations of the two components disagree with both sets of models (which agree very well between themselves).

The likely interpretation is that the temperatures from the model atmospheres place the components to the left of the evolutionary tracks, i.e., too warm. As discussed in $\S 4.3$, the model atmospheres are a significant source of the uncertainty in placing the components on Figure 15. A possible systematic error of only $\approx 100 \mathrm{~K}$ would be sufficient to resolve the discrepancy with the data. Therefore, while acquiring resolved spectra of the two components could help refine the temperature determination, the systematic uncertainties in the atmosphere models will still hamper accurate placement on the H-R diagram. We discuss this further in the next section.

The opposite interpretation is that the evolutionary models are incorrect, leading to $\mathrm{a} \approx 50 \%$ overprediction of the luminosities and $\mathrm{a} \approx 20 \%$ overprediction of the radii, given the component masses. Equivalently, if one were simply to assume that the H-R diagram positions are accurate, the evolutionary models would suggest individual masses of around 0.05 and $0.06 M_{\odot}$ from the Lyon and Tucson models, respectively, i.e., nearly a factor of 2 overestimate in the masses. While it may be that the evolutionary models are so substantially incorrect, such a conclusion is not compelling at this point, given the plausible errors in the $T_{\text {eff }}$ determinations. ${ }^{28}$

Direct mass determinations for the individual components from radial velocity monitoring and/or absolute orbital astrometry will help to further characterize the system. Such data will directly test the $q=0.936_{-0.008}^{+0.012}$ determined from the evolutionary tracks. The expected maximum radial velocity difference of the two components is only $4.6 \mathrm{~km} \mathrm{~s}^{-1}$. Since the two components are nearly equal mass and brightness, the orbital motion will be very difficult to detect in the integrated-light spectrum. Resolved AO spectroscopy will be required, and the small amplitude will make it a challenging measurement given the few kilometer per second accuracy that has been achieved for T dwarfs on the largest existing telescopes (Zapatero Osorio et al. 2007).

Individual mass measurements can in principle also test the evolutionary tracks directly. One can estimate the age of each component from its mass and luminosity (as we have done using the total mass) and see if the ages indicate coevality for the system. However, given the near-equal flux ratio of this system (and most ultracool binaries), this coevality test is unlikely to be very discriminating. Moreover, individual masses cannot resolve the discrepancy seen in the H-R diagram (Fig. 15), which largely arises from the uncertainties in the model atmospheres.

\subsection{Future Tests of Theory with Field Substellar Binaries}

With the advent of LGS AO on the largest ground-based telescopes, we can expect an increasing number of dynamical masses

\footnotetext{
${ }^{28}$ Note that a systematic error in our model-derived mass ratio cannot resolve the H-R diagram discrepancy. Since the ratio is basically unity, correcting any errors in $q$ would move one evolutionary track closer to one component, while the other track would move farther away from the other component. The evolutionary models could be brought into agreement with the data for component $\mathrm{A}$ for $q \lesssim 0.4$, but this is implausible given the nearly equal magnitudes of the two components. Such a small $q$ would also exacerbate the disagreement between the observations and the models for component $\mathrm{B}$.
} 
for ultracool field dwarfs in the near future. The most useful systems for testing theory will be those with both independent mass and age determinations, namely, binaries that are associated with open clusters/groups and/or field stars of known age. The former will present a significant technical challenge, e.g., ultracool binaries in the Hyades $(d=46.3 \pm 0.3 \mathrm{pc}$; Perryman et al. 1998) and Pleiades $(d=133.5 \pm 1.2 \mathrm{pc}$; Soderblom et al. 2005) with suitably short orbital periods are unresolvable with current technology and thus none are currently known. Ultracool binary companions to field stars are extremely rare, and thus while very valuable systems, these will only probe a very limited range of spectral type, age, and mass: only four systems are known with suitably short orbital periods $(P \lesssim 50 \mathrm{yr})$, the T1+T6 binary $\epsilon$ Ind Bab (McCaughrean et al. 2004), the L4+L4 binary HD 130948BC (Potter et al. 2002), the L4.5+L6 binary GJ 417BC (Bouy et al. 2003; Gizis et al. 2003), and the L4.5+L4.5 binary GJ 1001BC (Golimowski et al. $2004 b)$. Therefore, there is significant motivation to develop analyses that employ masses derived from the much more numerous field binaries. In this regard and as illustrated by our analysis for 2MASS J1534-2952AB, one can identify two orthogonal pathways to confront theory: (1) comparison to evolutionary tracks and (2) comparison to atmospheric models.

\subsubsection{Comparison to Evolutionary Tracks ("H-R Diagram Test")}

Direct measurements of $L_{\text {bol }}, T_{\text {eff }}$, and mass (or age) for brown dwarfs enable use of the H-R diagram, by comparing the observations to evolutionary tracks that correspond to the measured masses of the objects. As illustrated by Figure 15, the Lyon and Tucson tracks differ at the 5\%-10\% level in mass, and thus mass determinations of $2 \%-3 \%$ accuracy could discriminate between the two models, if $L_{\mathrm{bol}}$ and $T_{\mathrm{eff}}$ can be well measured. (Improvements in the parallaxes of many ultracool binaries will also be needed to achieve such accurate masses.) Accurate measurements for $L_{\text {bol }}$ are largely straightforward, as good as a few percent (e.g., $\S 3.2$ ). However, direct $T_{\text {eff }}$ determinations are extremely challenging, since radius measurements are needed. Brown dwarfs are too small and faint to be resolved with current or planned interferometers, and no eclipsing ultracool field binaries are yet known. Thus, $T_{\text {eff }}$ must be derived from modeling the observed colors, magnitudes, and/or spectra; the approach currently suffers from uncertainties at the level of a few to several hundred kelvin and systematic errors that are difficult to quantify (e.g., Cushing et al. 2008). In comparison, Figure 15 shows that $T_{\text {eff }}$ determinations good to $\lesssim 30 \mathrm{~K}$ are needed. Therefore, decisive tests of evolutionary tracks using field binaries will be challenged by this uncertainty in $T_{\text {eff }}$, in the absence of direct radius measurements.

\subsubsection{Comparison to Atmospheric Models ("Age/Mass Benchmark Test")}

Brown dwarfs obey a mass-luminosity-age $\left(M, L_{\mathrm{bol}}, t\right)$ relation, and for most field objects neither the mass nor the age is known. A commonly used approach to circumvent this limitation is to study brown dwarfs that are companions to main-sequence stars, where (indirect) age estimates are available from the primary star (e.g., Saumon et al. 2000; Geballe et al. 2001; McCaughrean et al. 2004; Metchev \& Hillenbrand 2006; Liu et al. 2007; Burgasser 2007a). This approach can also be applied to members of coeval clusters / groups and companions to post-main-sequence stars of known age (e.g., Kirkpatrick et al. 1999; Pinfield et al. 2006). In these situations, $L_{\mathrm{bol}}$ and $t$ are known, and combined with evolutionary models, one can derive $M$ and consequently $T_{\text {eff }}$ and $\log g$. Then the observed colors, magnitudes, and spectra can test the accuracy of atmospheric models with the same $T_{\text {eff }}$ and $\log g$. Examination of the known "age benchmark" $T$ dwarfs in this fashion finds that the properties deduced from atmospheric models are in good agreement with those from the evolutionary models, within the uncertainties in the ages and metallicities of the primary stars (Burgasser 2007a; Leggett et al. 2007a, 2008).

We suggest that, in an analogous fashion, field binaries with known masses can also serve as "benchmark" objects. In this case, $M$ and $L_{\mathrm{bol}}$ are known, and combined with evolutionary models, one can derive $t$, as we have done in $\S 4.1$. This provides $T_{\text {eff }}$ and $\log g$ and thereby allows tests of atmospheric models. The chain of analysis is identical to brown dwarf companions of known age: given independent knowledge of two quantities out of $\{M$, $\left.L_{\text {bol }}, t\right\}$, use evolutionary models to derive the third. ${ }^{29}$ In both cases, these benchmarks can also serve as anchor points for direct empirical calibration of spectroscopic diagnostics of $\log g$ and $T_{\text {eff }}$ (e.g., Pinfield et al. 2006; Burgasser et al. 2006a).

At face value, using objects that are age benchmarks or mass benchmarks is less fundamental than direct tests of the evolutionary models using the H-R diagram. However, in practice the Benchmark Test is much more feasible to implement and subject to much smaller systematic errors. In the absence of direct radius measurements, the H-R Diagram Test is held hostage to the systematic errors in the determination of $T_{\text {eff }}$ from atmospheric models. In contrast, the Benchmark Test relies on the evolutionary models, which are thought to be more robust (e.g., Chabrier et al. 2000). In short, given the choice of relying on atmospheric models (H-R Diagram Test) or evolutionary models (Benchmark Test), the evolutionary models are likely to be preferred.

To assess the relative utility of age benchmarks (brown dwarf companions to stars) compared to mass benchmarks (brown dwarfs with dynamical masses), we turn to equation (8). For an object with a measured $L_{\mathrm{bol}}$ and ignoring the weak dependence on $\kappa_{\mathrm{R}}$, given a measurement of $M$ or $t$ with accompanying uncertainty of $\delta M$ or $\delta t$, the fractional error in the remaining quantity is related by

$$
\frac{\delta t}{t}=2.03 \frac{\delta M}{M} .
$$

Typical uncertainties in the ages of main-sequence field stars are about 50\%-100\% (e.g., Kirkpatrick et al. 2001; Liu et al. 2007; Metchev \& Hillenbrand 2006), and thus age-benchmark objects would have a $25 \%-50 \%$ uncertainty in the mass inferred from evolutionary models. ${ }^{30}$ In contrast, dynamical masses of $\approx 5 \%-$ $10 \%$ accuracy will be possible in the next few years for many ultracool dwarfs, as we have already achieved with 2MASS J15342952AB, and hence mass-benchmark objects will have only a $\approx 10 \%-20 \%$ error in the age inferred from evolutionary models. So overall, age benchmarks can be expected to have $\{\delta t / t, \delta M / M\} \approx$ $\{50 \%-100 \%, 25 \%-50 \%\}$ while mass benchmarks would have uncertainties of order $\{\delta t / t, \delta M / M\} \approx\{10 \%-20 \%, 5 \%-10 \%\}$.

We can use the analytic fits to evolutionary models from Burrows et al. (2001) to gauge the relative accuracy on $T_{\text {eff }}$ and $\log g$ derived from both types of benchmarks. Using standard

\footnotetext{
29 The analogy between mass benchmarks and age benchmarks is an imperfect one, since brown dwarf companions also have metallicity determinations from their parent star, whereas field binaries do not. However, current studies of age benchmarks largely rely on evolutionary models computed for solar metallicity.

${ }^{30}$ Barnes (2007) finds that ages for solar-type stars derived from gyrochronology can have errors of only $15 \%-20 \%$.
} 

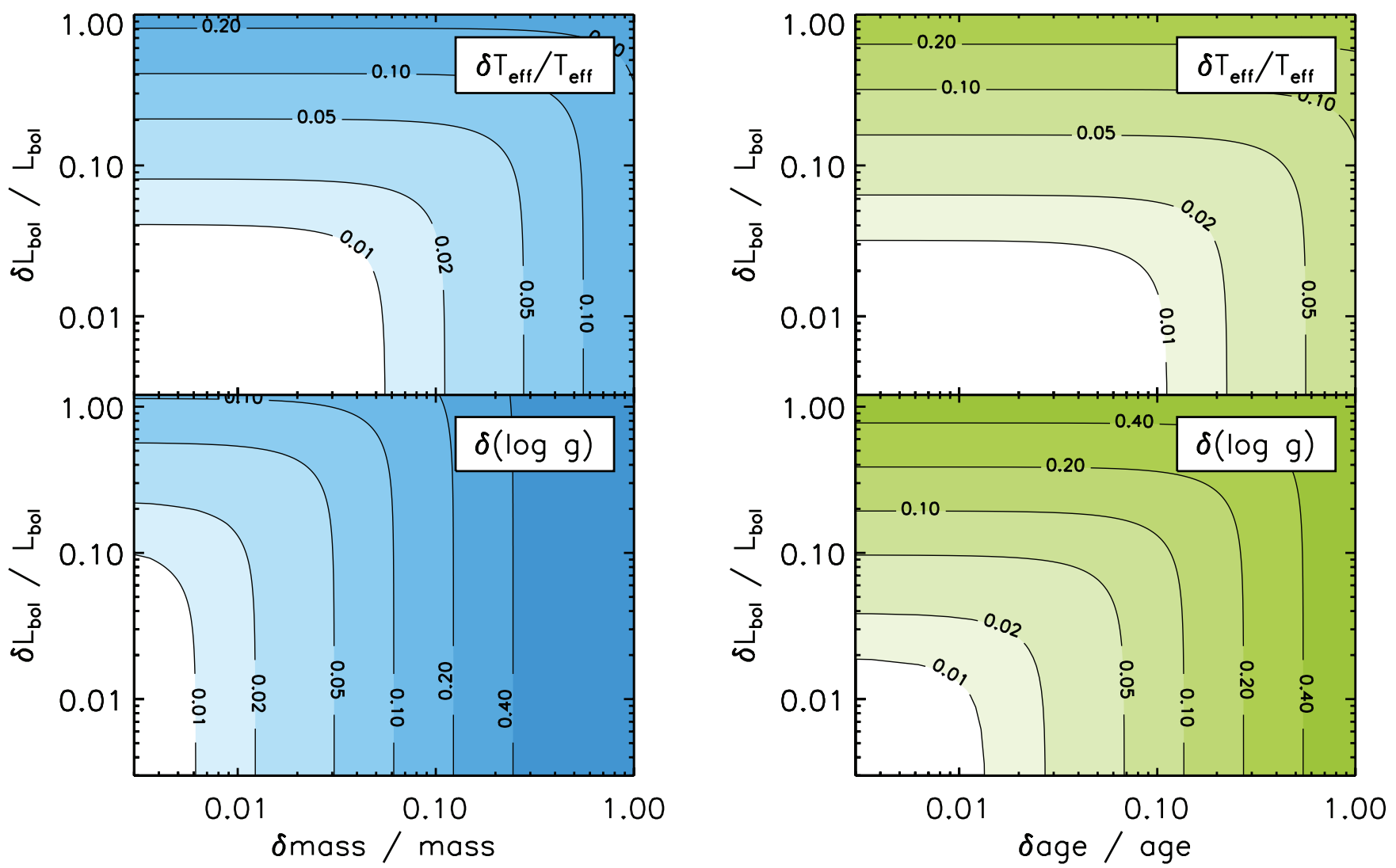

FIG. 16. - Contour plots showing the uncertainties in determining $T_{\text {eff }}$ and $\log g$ using mass benchmarks (brown dwarfs with dynamical masses) and age benchmarks

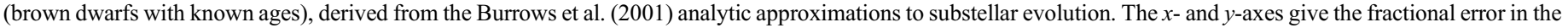
measurements of mass, age, and $L_{\mathrm{bol}}$, and the contours show the resulting fractional uncertainties in $T_{\text {eff }}$ and $\log g$ spaced from $1 \%$ to $40 \%$.

error propagation and assuming uncorrelated errors, we find for mass benchmarks

$$
\begin{gathered}
\frac{\delta T_{\mathrm{eff}}}{T_{\mathrm{eff}}}=\sqrt{\left(0.180 \frac{\delta M}{M}\right)^{2}+\left(0.246 \frac{\delta L_{\mathrm{bol}}}{L_{\mathrm{bol}}}\right)^{2}}, \\
\delta \log g=\sqrt{\left(1.63 \frac{\delta M}{M}\right)^{2}+\left(0.088 \frac{\delta L_{\mathrm{bol}}}{L_{\mathrm{bol}}}\right)^{2}}, \\
\delta T_{\text {eff }}=9 \mathrm{~K}\left(\frac{T_{\mathrm{eff}}}{1000 \mathrm{~K}}\right) \sqrt{\left(\frac{\delta M / M}{0.05}\right)^{2}+\left(2.73 \frac{\delta L_{\mathrm{bol}} / L_{\mathrm{bol}}}{0.10}\right)^{2}}, \\
\delta \log g=0.082 \mathrm{dex} \sqrt{\left(\frac{\delta M / M}{0.05}\right)^{2}+\left(1.08 \frac{\delta L_{\mathrm{bol}} / L}{0.10}\right)^{2}},
\end{gathered}
$$

where $\delta T_{\text {eff }}, \delta M, \delta L_{\mathrm{bol}}$, and $\delta \log g$ are the uncertainties in the temperature, mass, luminosity, and surface gravity, respectively. And then for age benchmarks, we find

$$
\begin{aligned}
\frac{\delta T_{\mathrm{eff}}}{T_{\mathrm{eff}}} & =\sqrt{\left(0.089 \frac{\delta t}{t}\right)^{2}+\left(0.314 \frac{\delta L_{\mathrm{bol}}}{L_{\mathrm{bol}}}\right)^{2}}, \\
\delta \log g & =\sqrt{\left(0.734 \frac{\delta t}{t}\right)^{2}+\left(0.517 \frac{\delta L_{\mathrm{bol}}}{L_{\mathrm{bol}}}\right)^{2}},
\end{aligned}
$$

$$
\begin{gathered}
\delta T_{\text {eff }}=44 \mathrm{~K}\left(\frac{T_{\mathrm{eff}}}{1000 \mathrm{~K}}\right) \sqrt{\left(\frac{\delta t / t}{0.5}\right)^{2}+\left(0.71 \frac{\delta L_{\mathrm{bol}} / L_{\mathrm{bol}}}{0.10}\right)^{2}}, \\
\delta \log g=0.37 \mathrm{dex} \sqrt{\left(\frac{\delta t / t}{0.5}\right)^{2}+\left(0.14 \frac{\delta L_{\mathrm{bol}} / L_{\mathrm{bol}}}{0.10}\right)^{2}} \cdot
\end{gathered}
$$

Thus, with representative values for the fractional errors in age $(50 \%)$, luminosity $(10 \%)$, and mass $(5 \%)$, we see that $T_{\text {eff }}$ and $\log g$ are better constrained by a factor of $\approx 5$ using mass benchmarks than age benchmarks. Figure 16 plots the derived analytic estimates for both types of benchmarks. These contour plots provide a convenient means to gauge the expected errors in $T_{\text {eff }}$ and $\log g$ determinations from benchmarks. The morphology of the contours also illustrates whether the observational errors in the age, mass, and/or $L_{\text {bol }}$ dominate the uncertainties in $T_{\text {eff }}$ and $\log g$. For the specific case of 2MASS J1534-2952AB, there is good agreement between the analytic estimates and the values derived directly from the actual evolutionary models (Table 5).

\section{CONCLUSIONS}

We have determined the first dynamical mass for a binary $\mathrm{T}$ dwarf, the T5.0+T5.5 system 2MASS J1534-2952AB, by combining six epochs of Keck LGS AO imaging from 2005 to 2008 with three epochs of HST imaging obtained in 2000 and 2006. Both data sets achieve milliarcsecond accuracy or better for the relative astrometry of the two components and are validated through extensive testing with images of simulated binaries. We employ a Markov chain Monte Carlo analysis to determine the 
orbital parameters and their uncertainties. The time baseline of our complete data set covers about half of the total period. We find that the orbital motion of the binary is viewed in an almost edge-on orientation and has a modest eccentricity. Our determination of a $15.1_{-1.6}^{+2.3} \mathrm{yr}$ orbital period is significantly longer than the original 4 yr estimate, as by chance the binary was at a very small projected separation when discovered in 2000.

The total mass of the system is $0.056 \pm 0.003 M_{\odot}\left(59 \pm 4 M_{\mathrm{J}}\right)$, including the uncertainty in the parallax. This is the second brown dwarf binary directly confirmed, the first among the field population. It is also the coolest and lowest mass binary with a dynamical mass determination to date.

With very accurate measurements of the total mass and the bolometric luminosity $\left[\log \left(L_{\text {bol }} / L_{\odot}\right)=-4.751 \pm 0.011\right]$, we use the Tucson and Lyon evolutionary tracks to determine the remaining physical properties for the system. The two sets of models give largely consistent results, which highlights the difficulty of distinguishing between them even with such precise observational data. We average the model results to represent the final determinations. We find a relatively youthful age for the system of $0.79 \pm 0.09 \mathrm{Gyr}(1 \sigma)$, consistent with its low tangential velocity relative to other field $\mathrm{T}$ dwarfs. The remaining physical parameters of the individual components are then fully determined: radii of $0.0986 \pm 0.0015$ and $0.0993 \pm 0.0017 R_{\odot}$, effective temperatures of $1028 \pm 17$ and $978 \pm 17 \mathrm{~K}$, surface gravities of $\log g=$ $4.91 \pm 0.04$ and $4.87 \pm 0.04$, and masses of $0.0287 \pm 0.0016 M_{\odot}$ $\left(30.1 \pm 1.7 M_{\mathrm{J}}\right)$ and $0.0269 \pm 0.0016 M_{\odot}\left(28.2 \pm 1.7 M_{\mathrm{J}}\right)$ for components $\mathrm{A}$ and $\mathrm{B}$, respectively. We take care to account for the covariances inherent in the measurement uncertainties, by using a Monte Carlo approach to derive these physical quantities from the evolutionary models. Our approach also assumes that the system is coeval and composed of only two components.

These precise determinations for 2MASS J1534-2952A and 2MASS J1534-2952B are in general accord with the $T_{\text {eff }}$ and $\log g$ values found previously for field $\mathrm{T}$ dwarfs based on model atmospheres and with the ages of $\mathrm{T}$ dwarfs predicted by Monte Carlo simulations of the solar neighborhood. However, on closer scrutiny, there are two potential discrepancies with past studies. Both suggest that the temperatures of field T dwarfs may be overestimated by $\approx 100 \mathrm{~K}$, although we stress that the two discrepancies must arise from independent effects: (1) The temperatures of 2MASS J1534-2952A and 2MASS J1534-2952B appear to be cooler than field objects of comparable spectral type. This resembles discrepancies previously noted by Metchev \& Hillenbrand (2006) and Luhman et al. (2007) for late L/early T dwarfs that are companions to young main-sequence stars. They have hypothesized that the effect is due to the gravity sensitivity of the L/T transition. The fact that this discrepancy also occurs for $2 \mathrm{MASS}$ J1534-2952AB suggests instead that the problem may arise from a factor of $\approx 6 \pm 3$ overestimate in the adopted ages of field objects when determining their temperatures using evolutionary tracks. Ages of $\sim 0.3-1.0 \mathrm{Gyr}$ are preferred based on this binary. (2) The temperatures of 2MASS J1534-2952A and 2MASS J15342952B are slightly cooler than inferred for other mid-T dwarfs from model atmospheres. Detailed analysis of the system's integratedlight and resolved spectra with model atmospheres is needed to directly assess the $T_{\text {eff }}$ and $\log g$ of the two components and to refine the comparison with the values derived from evolutionary models.

The positions of the two components on the H-R diagram are discrepant with theoretical evolutionary tracks corresponding to their individual masses. In fact, taken at face value, using the H-R diagram positions to infer masses from the evolutionary tracks would lead to masses of $\approx 0.05-0.06 M_{\odot}$, about a factor of 2 larger than the actual measured masses. While this discrepancy could stem from large systematic errors in the luminosities ( $\sim 50 \%$ errors) and/or radii ( $\sim 20 \%$ errors) predicted by evolutionary models, the likely cause is that temperatures from model atmospheres are too warm by $\approx 100 \mathrm{~K}$ for mid-T dwarfs. This highlights the need for continued improvements to the model atmospheres.

Future monitoring of 2MASS J1534-2952AB will help to refine its orbit and its dynamical mass. The orbital separation of the system is now rapidly decreasing and will not be readily resolvable again until around 2011. At the same time, an improved parallax for the system will be required: the uncertainty in the total mass from the orbit fitting is 3\%, compared to the $5 \%$ that arises from the uncertainty in the parallax. Radial velocity monitoring and/or absolute astrometry will directly determine the individual masses and test if the system is a higher order multiple. However, given the very similar fluxes of the two components (implying nearly equal mass), individual mass measurements are unlikely to resolve the discordant H-R diagram position of the two components relative to evolutionary tracks. This problem is likely driven by the systematic uncertainties in current model atmospheres for $\mathrm{T}$ dwarfs.

The fundamental characteristic of the field population is that it spans a range of (largely unknown) ages. However, despite this uncertainty, field brown dwarf binaries can strongly test theoretical models, if analyzed appropriately. These systems will be especially valuable in light of the current paucity of eclipsing field ultracool binaries and resolvable, short-period ultracool binaries in open clusters/groups. ${ }^{31}$ Specifically, attempts to directly test different evolutionary tracks by placing ultracool objects on the H-R diagram (the H-R Diagram Test) will be challenging, given the similarity between the tracks and the difficulty in independently determining $T_{\text {eff }}$ with model atmospheres. Instead, atmosphere models can be confronted against $\log g$ and $T_{\text {eff }}$ values for ultracool objects as derived from the evolutionary models, which can be exceptionally precise (the Benchmark Test). This approach has previously been applied to single brown dwarfs that are companions to stars of known age (age benchmarks). We suggest that in an analogous fashion, field ultracool binaries with dynamical mass determinations (mass benchmarks) can test the model atmospheres. In fact, given the plausible observational uncertainties, mass benchmarks are likely to provide stronger constraints (by a factor of $\approx 5$ ) on $\log g$ and $T_{\text {eff }}$ than age benchmarks, since dynamical masses can be determined far more accurately than ages for main-sequence field stars. With the widespread advent of LGS AO on the largest ground-based telescopes, we can look forward to a rapid increase in dynamical mass determinations for low-mass field binaries and thus substantial advances in our understanding of the properties and evolution of substellar objects.

We gratefully acknowledge the Keck LGS AO team for their exceptional efforts in bringing the LGS AO system to fruition. It is a pleasure to thank Antonin Bouchez, David LeMignant, Marcos van Dam, Randy Campbell, Al Conrad, Jim Lyke, Hien Tran, Robert LaFon, Kenny Graves, Cindy Wilburn, Joel Aycock, Terry Stickel, Gary Punawai, and the Keck Observatory staff for assistance with the observations. We thank Alan Stockton for a fortuitous swap of observing nights in spring 2005, Brian Cameron for sharing his NIRC2 instrumental distortion analysis, Hai Fu for IDL plotting assistance, Michael Cushing and Mark Pitts for assistance with the IRTF/SpeX spectroscopy, Adam Burrows

\footnotetext{
${ }^{31}$ For reference, about 1 out of 1000 stars is an eclipsing binary, whereas there are only $\sim 600 \mathrm{~L}$ and $\mathrm{T}$ dwarfs known.
} 
and Isabelle Baraffe for finely gridded evolutionary tracks, and Thierry Forveille and Adam Burgasser for careful readings of the manuscript. We have benefitted from enlightening discussions with Michael Cushing, Adam Burrows, and Isabelle Baraffe about substellar models, Thierry Forveille about low-mass binaries, Brian Cameron about astrometry with NIRC2, and Jay Anderson about astrometry with HST. Our research has employed the 2MASS data products; NASA's Astrophysical Data System; the SIMBAD database operated at CDS, Strasbourg, France; the M, L, and $\mathrm{T}$ dwarf compendium housed at DwarfArchives.org and maintained by Chris Gelino, Davy Kirkpatrick, and Adam Burgasser (Kirkpatrick 2003; Gelino et al. 2004); and the SpeX Prism Spectral Libraries maintained by Adam Burgasser at http://www .browndwarfs.org/spexprism. M. C. L. and T. J. D. acknowledge support for this work from NSF grant AST 05-07833 and an Alfred P. Sloan Research Fellowship. M. J. I. acknowledges Michelson Fellowship support from the Michelson Science Center and the NASA Navigator Program. The United Kingdom Infrared Telescope (UKIRT) is operated by the Joint Astronomy Centre on behalf of the Science and Technology Facilities Council of the UK. Finally, the authors wish to recognize and acknowledge the very significant cultural role and reverence that the summit of Mauna Kea has always had within the indigenous Hawaiian community. We are most fortunate to have the opportunity to conduct observations from this mountain.

Facilities: Keck:II (LGS AO, NIRC2), HST (WFPC2, ACS), IRTF (SpeX)
Allen, P. R. 2007, ApJ, 668, 492

Allen, P. R., Koerner, D. W., Reid, I. N., \& Trilling, D. E. 2005, ApJ, 625, 385

Anderson, J., \& King, I. R. 1999, PASP, 111, 1095 2003, PASP, 115,113

2004, ACS Instrument Science Rep. 2004-15

Baraffe, I., Chabrier, G., Barman, T. S., Allard, F., \& Hauschildt, P. H. 2003, A\&A, 402, 701

Barnes, S. A. 2007, ApJ, 669, 1167

Basri, G., \& Reiners, A. 2006, AJ, 132, 663

Bouy, H., Brandner, W., Martín, E. L., Delfosse, X., Allard, F., \& Basri, G. 2003, AJ, 126, 1526

Bouy, H., et al. 2004, A\&A, 423, 341

2008, A\&A, 481, 757

Bremaud, P. 1999, Markov Chains: Gibbs Fields, Monte Carlo Simulation, and Queues (New York: Springer)

Burgasser, A. J. 2002, Ph.D. thesis, Caltech

2004, ApJS, 155, 191

2007a, ApJ, 658, 617

2007b, ApJ, 659, 655

Burgasser, A. J., Burrows, A., \& Kirkpatrick, J. D. 2006a, ApJ, 639, 1095

Burgasser, A. J., Geballe, T. R., Leggett, S. K., Kirkpatrick, J. D., \& Golimowski, D. A. 2006b, ApJ, 637, 1067

Burgasser, A. J., Kirkpatrick, J. D., Cruz, K. L., Reid, I. N., Leggett, S. K., Liebert, J., Burrows, A., \& Brown, M. E. 2006c, ApJS, 166, 585

Burgasser, A. J., Kirkpatrick, J. D., Liebert, J., \& Burrows, A. 2003a, ApJ, 594, 510

Burgasser, A. J., Kirkpatrick, J. D., \& Lowrance, P. J. 2005a, AJ, 129, 2849

Burgasser, A. J., Kirkpatrick, J. D., Reid, I. N., Brown, M. E., Miskey, C. L., \& Gizis, J. E. 2003b, ApJ, 586, 512

Burgasser, A. J., McElwain, M. W., Kirkpatrick, J. D., Cruz, K. L., Tinney, C. G., \& Reid, I. N. 2004, AJ, 127, 2856

Burgasser, A. J., Reid, I. N., Leggett, S. K., Kirkpatrick, J. D., Liebert, J., \& Burrows, A. 2005b, ApJ, 634, L177

Burgasser, A. J., Reid, I. N., Siegler, N., Close, L., Allen, P., Lowrance, P., \& Gizis, J. 2007, in Protostars and Planets V, ed. B. Reipurth, D. Jewitt, \& K. Keil (Tucson: Univ. Arizona Press), 427

Burgasser, A. J., et al. 2000, AJ, 120, 1100

. 2002, ApJ, 564, 421

Burrows, A., Burgasser, A. J., Kirkpatrick, J. D., Liebert, J., Milsom, J. A., Sudarsky, D., \& Hubeny, I. 2002, ApJ, 573, 394

Burrows, A., Hubbard, W. B., Lunine, J. I., \& Liebert, J. 2001, Rev. Mod. Phys., 73, 719

Burrows, A., Sudarsky, D., \& Hubeny, I. 2006, ApJ, 640, 1063

Burrows, A., et al. 1997, ApJ, 491, 856

Chabrier, G., Baraffe, I., Allard, F., \& Hauschildt, P. 2000, ApJ, 542, 464

Chiu, K., et al. 2006, AJ, 131, 2722

Close, L. M., Siegler, N., Freed, M., \& Biller, B. 2003, ApJ, 587, 407

Cohen, J. G., \& Cromer, J. 1988, PASP, 100, 1582

Cushing, M. C., Vacca, W. D., \& Rayner, J. T. 2004, PASP, 116, 362

Cushing, M. C., et al. 2006, ApJ, 648, 614 2008, ApJ, 678, 1372

Dahn, C. C., et al. 2002, AJ, 124, 1170

Delorme, P., et al. 2008, A\&A, 482, 961

Diolaiti, E., Bendinelli, O., Bonaccini, D., Close, L., Currie, D., \& Parmeggiani, G. 2000, A\&AS, 147, 335

Duquennoy, A., \& Mayor, M. 1991, A\&A, 248, 485

Fischer, D. A., \& Marcy, G. W. 1992, ApJ, 396, 178

Ford, E. B. 2005, AJ, 129, 1706
Forveille, T., et al. 1999, A\&A, 351, 619

Foy, R., \& Labeyrie, A. 1985, A\&A, 152, L29

Geballe, T., et al. 2002, ApJ, 564, 466

Geballe, T. R., Saumon, D., Leggett, S. K., Knapp, G. R., Marley, M. S., \& Lodders, K. 2001, ApJ, 556, 373

Gelino, C. R., Kirkpatrick, J. D., \& Burgasser, A. J. 2004, BAAS, 36, 1354

Gizis, J. E., \& Reid, I. N. 2006, AJ, 131, 638

Gizis, J. E., Reid, I. N., Knapp, G. R., Liebert, J., Kirkpatrick, J. D., Koerner,

D. W., \& Burgasser, A. J. 2003, AJ, 125, 3302

Golimowski, D. A., et al. 2004a, AJ, 127, 3516

2004b, AJ, 128, 1733

Gregory, P. C. 2005, ApJ, 631, 1198

Happer, W., MacDonald, G. J., Max, C. E., \& Dyson, F. J. 1994, Opt. Soc. Am. J. A, 11,263

Hayashi, C., \& Nakano, T. 1963, Prog. Theor. Phys., 30, 460

Holtzman, J. A. et al. 1995, PASP, 107, 156

Ireland, M., Kraus, A., Martinache, F., Lloyd, J. P., \& Tuthill, P. G. 2008, ApJ, 678,463

Kenworthy, M., et al. 2001, ApJ, 554, L67

Kirkpatrick, J. D. 2003, in IAU Symp. 211, Brown Dwarfs, ed. E. Martin (San Francisco: ASP), 189 2005, ARA\&A, 43, 195

Kirkpatrick, J. D., Allard, F., Bida, T., Zuckerman, B., Becklin, E. E., Chabrier, G., \& Baraffe, I. 1999, ApJ, 519, 834

Kirkpatrick, J. D., Dahn, C. C., Monet, D. G., Reid, I. N., Gizis, J. E., Liebert, J., \& Burgasser, A. J. 2001, AJ, 121, 3235

Kirkpatrick, J. D., et al. 2000, AJ, 120, 447

Knapp, G. R., et al. 2004, AJ, 127, 3553

Konopacky, Q. M., Ghez, A. M., Duchêne, G., McCabe, C., \& Macintosh, B. A. 2007, AJ, 133, 2008

Krist, J. 1995, in ASP Conf. Ser. 77, Astronomical Data Analysis Software and Systems IV, ed. R. A. Shaw, H. E. Payne, \& J. J. E. Hayes (San Francisco: ASP), 349

Kumar, S. S. 1963, ApJ, 137, 1121

Lane, B. F., Zapatero Osorio, M. R., Britton, M. C., Martín, E. L., \& Kulkarni, S. R. 2001, ApJ, 560, 390

Leggett, S. K., Marley, M. S., Freedman, R., Saumon, D., Liu, M. C., Geballe, T. R., Golimowski, D. A., \& Stephens, D. C. 2007a, ApJ, 667, 537

Leggett, S. K., Saumon, D., Marley, M. S., Geballe, T. R., Golimowski, D. A., Stephens, D., \& Fan, X. 2007b, ApJ, 655, 1079

Leggett, S. K., et al. 2008, ApJ, 682, 1256

Liebert, J., \& Burgasser, A. J. 2007, ApJ, 655, 522

Liu, M. C. 2006, Proc. SPIE, 6272, 14

Liu, M. C., \& Leggett, S. K. 2005, ApJ, 634, 616

Liu, M. C., Leggett, S. K., \& Chiu, K. 2007, ApJ, 660, 1507

Liu, M. C., Leggett, S. K., Golimowski, D. A., Chiu, K., Fan, X., Geballe, T. R., Schneider, D. P., \& Brinkmann, J. 2006, ApJ, 647, 1393

Lloyd, J. P., Martinache, F., Ireland, M. J., Monnier, J. D., Pravdo, S. H., Shaklan, S. B., \& Tuthill, P. G. 2006, ApJ, 650, L131

Looper, D. L., Kirkpatrick, J. D., \& Burgasser, A. J. 2007, AJ, 134, 1162

Luhman, K. L., et al. 2007, ApJ, 654, 570

Martín, E. L., Koresko, C. D., Kulkarni, S. R., Lane, B. F., \& Wizinowich, P. L. 2000, ApJ, 529, L37

Maxted, P. F. L., \& Jeffries, R. D. 2005, MNRAS, 362, L45

McCaughrean, M. J., Close, L. M., Scholz, R.-D., Lenzen, R., Biller, B., Brandner, W., Hartung, M., \& Lodieu, N. 2004, A\&A, 413, 1029

Metchev, S., Kirkpatrick, J. D., Berriman, G. B., \& Looper, D. 2008, ApJ, 676, 1281 
Metchev, S. A., \& Hillenbrand, L. A. 2006, ApJ, 651, 1166

Monet, D. G., Dahn, C. C., Vrba, F. J., Harris, H. C., Pier, J. R., Luginbuhl, C. B., \& Ables, H. D. 1992, AJ, 103, 638

Monet, D. G., et al. 2003, AJ, 125, 984

Nordström, B., et al. 2004, A\&A, 418, 989

Oppenheimer, B. R., Kulkarni, S. R., Matthews, K., \& Nakajima, T. 1995, Science, 270, 1478

Pascu, D., et al. 1998, AJ, 115, 1190 2004, AJ, 127, 2988

Patten, B. M., et al. 2006, ApJ, 651, 502

Perryman, M. A. C., et al. 1998, A\&A, 331, 81

Pinfield, D. J., Jones, H. R. A., Lucas, P. W., Kendall, T. R., Folkes, S. L., Day-Jones, A. C., Chappelle, R. J., \& Steele, I. A. 2006, MNRAS, 368, 1281

Potter, D., Martín, E. L., Cushing, M. C., Baudoz, P., Brandner, W., Guyon, O., \& Neuhäuser, R. 2002, ApJ, 567, L133

Pravdo, S. H., Shaklan, S. B., Wiktorowicz, S. J., Kulkarni, S., Lloyd, J. P., Martinache, F., Tuthill, P. G., \& Ireland, M. J. 2006, ApJ, 649, 389

Press, W. H., Teukolsky, S. A., Vetterling, W. T., \& Flannery, B. P. 1992, Numerical Recipes in C: The Art of Scientific Computing (2nd ed.; Cambridge: Cambridge Univ. Press)

Rayner, J. T., Toomey, D. W., Onaka, P. M., Denault, A. J., Stahlberger, W. E., Watanabe, D. Y., \& Wang, S. 1998, Proc. SPIE, 3354, 468

Reach, W. T., et al. 2005, PASP, 117, 978

Reid, I. N., Cruz, K. L., Burgasser, A. J., \& Liu, M. C. 2008, AJ, 135, 580

Reid, I. N., Gizis, J. E., Kirkpatrick, J. D., \& Koerner, D. W. 2001, AJ, 121, 489

Saumon, D., Bergeron, P., Lunine, J. I., Hubbard, W. B., \& Burrows, A. 1994, ApJ, 424, 333
Saumon, D., Geballe, T. R., Leggett, S. K., Marley, M. S., Freedman, R. S., Lodders, K., Fegley, B., Jr., \& Sengupta, S. K. 2000, ApJ, 541, 374

Saumon, D., et al. 2007, ApJ, 656, 1136

Simon, M., Bender, C., \& Prato, L. 2006, ApJ, 644, 1183

Simons, D. A., \& Tokunaga, A. 2002, PASP, 114, 169

Soderblom, D. R., Nelan, E., Benedict, G. F., McArthur, B., Ramirez, I., Spiesman, W., \& Jones, B. F. 2005, AJ, 129, 1616

Stassun, K. G., Mathieu, R. D., \& Valenti, J. A. 2006, Nature, 440, 311

Stephens, D. C., \& Leggett, S. K. 2004, PASP, 116, 9

Stone, R. C. 1984, A\&A, 138, 275

Tegmark, M., et al. 2004, Phys. Rev. D, 69, 103501

Thompson, L. A., \& Gardner, C. S. 1987, Nature, 328, 229

Tinney, C. G., Burgasser, A. J., \& Kirkpatrick, J. D. 2003, AJ, 126, 975

Tinney, C. G., Burgasser, A. J., Kirkpatrick, J. D., \& McElwain, M. W. 2005, AJ, 130, 2326

Tokunaga, A. T., Simons, D. A., \& Vacca, W. D. 2002, PASP, 114, 180

Torres, G. 1999, PASP, 111, 169

Vacca, W. D., Cushing, M. C., \& Rayner, J. T. 2003, PASP, 115, 389

van Dam, M. A., et al. 2006, PASP, 118, 310

Vrba, F. J., et al. 2004, AJ, 127, 2948

Warren, S. J., et al. 2007, MNRAS, 381, 1400

Wielen, R. 1977, A\&A, 60, 263

Wizinowich, P. L., et al. 2006, PASP, 118, 297

Zapatero Osorio, M. R., Lane, B. F., Pavlenko, Y., Martín, E. L., Britton, M., \& Kulkarni, S. R. 2004, ApJ, 615, 958

Zapatero Osorio, M. R., Martín, E. L., Béjar, V. J. S., Bouy, H., Deshpande, R., \& Wainscoat, R. J. 2007, ApJ, 666, 1205

Note added in proof.-A. Ghez et al. (ApJ, in press; arXiv:0808.2870) have recently determined a value for the P. A. of the $+y$-axis of NIRC2 that is identical but opposite in sign to the value we used from Pravdo et al. (2006). To assess the effect of this difference, we refit our astrometry with the Ghez et al. calibration, which would increase the sky P. A. values in Table 1 by $+0.26^{\circ}$ for the Keck measurements. The result is that the orbital parameters change by $\lesssim 0.1 \sigma$ except for the P. A. of the ascending node $(\Omega)$, which increases by $0.26^{\circ}(0.7 \sigma)$ as expected. The uncertainties in the orbital parameters change by $\lesssim 10 \%$, and the reduced $\chi^{2}$ of the best-fitting orbit increases from 0.9 to 1.0 . 